\title{
THEORY OF VALUATIONS ON MANIFOLDS, III. MULTIPLICATIVE STRUCTURE IN THE GENERAL CASE
}

\author{
SEMYON ALESKER AND JOSEPH H. G. FU
}

\begin{abstract}
This is the third part of a series of articles where the theory of valuations on manifolds is constructed. In the second part of this series the notion of a smooth valuation on a manifold was introduced. The goal of this article is to put a canonical multiplicative structure on the space of smooth valuations on general manifolds, thus extending some of the affine constructions from the first author's 2004 paper and, from the first part of this series.
\end{abstract}

\section{INTRODUCTION}

In convexity there are many geometrically interesting and well known examples of valuations on convex sets, including Lebesgue measure, the Euler characteristic, surface area, mixed volumes, and affine surface area. For a description of older classical developments on this subject we refer to the surveys [17], [16]. For general background on convexity we refer to the book [18].

Approximately during the last decade there has been significant progress in this classical subject, leading to new classification results for various classes of valuations, and to the discovery of new structures on them. This progress has shed new light on the notion of valuation, permitting an extension to the more general setting of valuations on manifolds, and to sets which are not necessarily convex (a concept which in any case has no meaning on a general manifold). The development of the theory of valuations on manifolds was started by one of the authors in the first two parts [4], [5] of the present series of articles.

In [5] the notion of smooth valuation on a smooth manifold was introduced. Roughly put a smooth valuation can be thought of as a finitely additive $\mathbb{C}$-valued measure on a class of nice subsets; this measure is required to satisfy some additional assumptions of continuity (or rather smoothness) in some sense. The basic examples of smooth valuations on a general manifold $X$ are smooth measures on $X$ and the Euler characteristic. Moreover the well known intrinsic volumes of convex sets can be generalized to provide examples of smooth valuations on an arbitrary Riemannian manifold; these valuations are known as Lipschitz-Killing curvatures.

The goal of this article is to put a canonical multiplicative structure on the space of smooth valuations on a general smooth manifold. When the manifold is an affine

Received by the editors October 21, 2005 and, in revised form, December 2, 2005.

2000 Mathematics Subject Classification. Primary 52B45, 52A39, 53C65.

The first author was partially supported by ISF grant 1369/04.

The second author was partially supported by NSF grant DMS-0204826. 
space the multiplicative structure was defined in [4] (in the more specific situation of valuations polynomial with respect to translations it was defined in [3]). The construction of the product on general manifolds presented in this article uses the construction from [4] for the affine case in combination with tools from geometric measure theory. Roughly it works as follows. Choosing a coordinate atlas for $X$, one uses the product of valuations on $\mathbb{R}^{n}$, defined by the construction of [4], to define the product locally. Then one shows that the products obtained on each coordinate patch coincide on pairwise intersections, and that the result does not depend on the choice of atlas. This step uses geometric measure theory.

Let us denote by $V^{\infty}(X)$ the space of smooth valuations on $X$. The product

$$
V^{\infty}(X) \times V^{\infty}(X) \rightarrow V^{\infty}(X)
$$

defined in this article is a continuous map, with respect to which $V^{\infty}(X)$ becomes a commutative associative algebra with unit (where the unit is the Euler characteristic).

In [5] it was shown that the assignment to any open subset $U \subset X$

$$
U \mapsto V^{\infty}(U),
$$

with the natural operations of restriction, defines a sheaf of vector spaces on $X$ denoted by $\mathcal{V}_{X}^{\infty}$. The multiplicative structure on smooth valuations defined in this article commutes with restriction to open subsets. Hence $\mathcal{V}_{X}^{\infty}$ becomes a sheaf of commutative associative algebras with unit. Further properties of the multiplicative structure are studied in the fourth part of this series [6].

The article is organized as follows. Section 1 contains some background; it does not contain new results. There we also fix some notation used throughout the article. In Section 2 we discuss normal cycles; the exposition follows mostly [10][13]. Then we explain how to use normal cycles to construct valuations. We also recall related results from geometric measure theory. In Section 3 we prove some auxiliary results of a technical nature. The main part of the article is Section 4, where we present a construction of the product on smooth valuations and prove that it is independent of the choices involved.

\section{BACKGROUND}

In Subsection 1.1 we fix some notation which will be used throughout the article. In Subsection 1.2 we recall some results from [1] and deduce from them Corollary 1.2.2, which will be used later on. In Subsection 1.3 we recall some necessary facts from the theory of valuations and some results from [4] and [5].

1.1. Notation. Let $V$ be a finite dimensional real vector space.

- Let $\mathcal{K}(V)$ denote the family of convex compact subsets of $V$.

- Let $\mathbb{R}_{\geq 0}$ denote the the set of non-negative real numbers.

- For a manifold $X$ let us denote by $\left|\omega_{X}\right|$ (or just by $|\omega|$ if it does not lead to confusion) the line bundle of densities over $X$.

- For a smooth manifold $X$ let $\mathcal{P}(X)$ denote the family of all simple subpolyhedra of $X$. (Namely $P \in \mathcal{P}(X)$ iff $P$ is a compact subset of $X$ locally diffeomorphic to $\mathbb{R}^{k} \times \mathbb{R}_{>0}^{n-k}$ for some $0 \leq k \leq n$. For a precise definition see [5], Subsection 2.1.)

- We denote by $\mathbb{P}_{+}(V)$ the oriented projectivization of $V$. Namely $\mathbb{P}_{+}(V)$ is the manifold of oriented lines in $V$ passing through the origin. 
- For a convex compact set $A \in \mathcal{K}(V)$ let us denote by $h_{A}$ the supporting functional of $A, h_{A}: V^{*} \rightarrow \mathbb{R}$. It is defined by

$$
h_{A}(y):=\sup \{y(x) \mid x \in A\} .
$$

- Let $L$ denote the (real) line bundle over $\mathbb{P}_{+}\left(V^{*}\right)$ such that its fiber over an oriented line $l \in \mathbb{P}_{+}\left(V^{*}\right)$ is equal to the dual line $l^{*}$.

- A subset $A$ of a Euclidean space $V$ is said to have positive reach (or to be semi-convex) if there exists $\varepsilon>0$ such that for any $x \in V$ with dist $(x, A)<\varepsilon$ there exists a unique point $y \in A$ with $\operatorname{dist}(x, y)=\operatorname{dist}(x, A)$ (cf. [8]). The reach of $A$ is defined to be the supremum of all such $\varepsilon$. Note that $A$ is convex iff $\operatorname{reach}(A)=\infty$.

1.2. Some convexity. First let us recall some results from [1]. Let $\bar{K}=\left(K_{1}, K_{2}\right.$, $\left.\ldots, K_{s}\right)$ be an $s$-tuple of compact convex subsets of $V$. Let $r \in \mathbb{N} \cup\{\infty\}$. For any $\mu \in$ $C^{r}\left(V,\left|\omega_{V}\right|\right)$ consider the function $M_{\bar{K}} F: \mathbb{R}_{+}^{s} \rightarrow \mathbb{C}$, where $\mathbb{R}_{+}^{s}=\left\{\left(\lambda_{1}, \ldots, \lambda_{s}\right) \mid \lambda_{j} \geq\right.$ $0\}$, defined by

$$
\left(M_{\bar{K}} \mu\right)\left(\lambda_{1}, \ldots, \lambda_{s}\right)=\mu\left(\sum_{i=1}^{s} \lambda_{i} K_{i}\right) .
$$

1.2.1. Theorem ([1]). (1) $M_{\bar{K}} \mu \in C^{r}\left(\mathbb{R}_{+}^{s}\right)$ and $M_{\bar{K}}$ is a continuous operator from $C^{r}\left(V,\left|\omega_{V}\right|\right)$ to $C^{r}\left(\mathbb{R}_{+}^{s}\right)$.

(2) Assume that a sequence $\mu^{(m)}$ converges to $\mu$ in $C^{r}\left(V,\left|\omega_{V}\right|\right)$. Let $K_{i}^{(m)}, K_{i}, i$ $=1, \ldots, s, m \in \mathbb{N}$, be convex compact sets in $V$, and for every $i=1, \ldots, s, K_{i}^{(m)} \rightarrow$ $K_{i}$ in the Hausdorff metric as $m \rightarrow \infty$. Then $M_{\bar{K}^{(m)}} \mu^{(m)} \rightarrow M_{\bar{K}} \mu$ in $C^{r}\left(\mathbb{R}_{+}^{s}\right)$ as $m \rightarrow \infty$.

1.2.2. Corollary. Let $r \in \mathbb{N}$. Let $R>0$. Then there exists a constant $C$ depending on $r, R$, and $n$ only such that for any $\mu \in C^{r}\left(V,\left|\omega_{V}\right|\right)$ and any $K \in \mathcal{K}(V)$ contained in a centered Euclidean ball of radius $R$ one has

$$
\left|\frac{\partial^{r}}{\partial \lambda_{1} \ldots \partial \lambda_{r}}\right|{ }_{0} \mu\left(K+\sum_{i=1}^{r} \lambda_{i} A_{i}\right) \mid \leq C \cdot\|\mu\|_{C^{r}(C R \cdot D)} \cdot \prod_{i=1}^{r}\left\|h_{A_{i}}\right\|_{C^{2}\left(\mathbb{P}_{+}\left(V^{*}\right)\right)} .
$$

Proof. Consider the functional

$$
\psi: C^{r}\left(V,\left|\omega_{V}\right|\right) \times \mathcal{K}(V)^{r+1} \rightarrow \mathbb{C}
$$

given by

$$
\psi\left(\mu ; K ; A_{1}, \ldots, A_{r}\right)=\left.\frac{\partial^{r}}{\partial \lambda_{1} \ldots \partial \lambda_{r}}\right|_{0} \mu\left(K+\sum_{i=1}^{r} \lambda_{i} A_{i}\right) .
$$

By Theorem 1.2.1 $\psi$ is a continuous map. Clearly $\psi$ is linear with respect to $\mu$ and symmetric with respect to $A_{1}, \ldots, A_{r}$. Moreover $\psi$ is Minkowski additive with respect to each of $A_{1}, \ldots, A_{r}$. (Minkowski additivity, say with respect to $A_{1}$, means that

$$
\begin{aligned}
\psi\left(\mu ; K ; \alpha A_{1}^{\prime}+\beta A_{1}^{\prime \prime}, A_{2}, \ldots, A_{r}\right)=\alpha \psi & \left(\mu ; K ; A_{1}^{\prime}, A_{2}, \ldots, A_{r}\right) \\
& +\beta \psi\left(\mu ; K ; A_{1}^{\prime \prime}, A_{2}, \ldots, A_{r}\right)
\end{aligned}
$$

for any $\alpha, \beta \geq 0, A_{1}^{\prime}, A_{1}^{\prime \prime} \in \mathcal{K}(V)$.) 
Let $h \in C^{2}\left(\mathbb{P}_{+}\left(V^{*}\right), L\right)$. Then $h$ can be presented as

$$
h=h_{A^{\prime}}-h_{A^{\prime \prime}}
$$

where $h_{A^{\prime}}, h_{A^{\prime \prime}} \in C^{2}\left(\mathbb{P}_{+}\left(V^{*}\right), L\right)$ are supporting functionals of convex compact sets $A^{\prime}, A^{\prime \prime} \in \mathcal{K}(V)$ respectively, and

$$
\max \left\{\left\|h_{A^{\prime}}\right\|_{C^{2}\left(\mathbb{P}_{+}\left(V^{*}\right)\right)},\left\|h_{A^{\prime \prime}}\right\|_{C^{2}\left(\mathbb{P}_{+}\left(V^{*}\right)\right)}\right\} \leq c\|h\|_{C^{2}\left(\mathbb{P}_{+}\left(V^{*}\right)\right)}
$$

where $c$ is a constant depending on $n$ only. Indeed let us take $h_{A^{\prime}}=h+T \cdot h_{D}, A^{\prime \prime}=$ $T \cdot D$ where $D$ is the unit Euclidean ball, and $T>0$ is a large enough constant depending on $\|h\|_{C^{2}\left(\mathbb{P}_{+}\left(V^{*}\right)\right)}$. Now let us extend $\psi$ to a functional

$$
\tilde{\psi}: C^{r}\left(V,\left|\omega_{V}\right|\right) \times \mathcal{K}(V) \times\left(C^{2}\left(\mathbb{P}_{+}\left(V^{*}\right), L\right)\right)^{r} \rightarrow \mathbb{C}
$$

by linearity. More precisely let

$$
\left(\mu ; K ; h_{1}, \ldots, h_{r}\right) \in C^{r}\left(V,\left|\omega_{V}\right|\right) \times \mathcal{K}(V) \times\left(C^{2}\left(\mathbb{P}_{+}\left(V^{*}\right), L\right)\right)^{r} .
$$

Let us define $\tilde{\psi}\left(\mu ; K ; h_{1}, \ldots, h_{r}\right)$ recursively on the number of non-convex functions among $h_{1}, \ldots, h_{r}$. If this number is equal to zero, there is nothing to define. Assume we have defined $\tilde{\psi}$ for $k-1<r$ not necessarily convex functions. Let us define it for $k$ such functions. We may assume that $h_{i}, i>k$ are convex. Choose a presentation

$$
h_{k}=h_{A_{k}^{\prime}}-h_{A_{k}^{\prime \prime}}
$$

as in (1), and satisfying (2). Now define

$$
\begin{array}{r}
\tilde{\psi}\left(\mu ; K ; h_{1}, \ldots, h_{k-1}, h_{k}, h_{k+1}, \ldots, h_{r}\right)= \\
\tilde{\psi}\left(\mu ; K ; h_{1}, \ldots, h_{k-1}, h_{A_{k}^{\prime}}, h_{k+1}, \ldots, h_{r}\right)-\tilde{\psi}\left(\mu ; K ; h_{1}, \ldots, h_{k-1}, h_{A_{k}^{\prime \prime}}, h_{k+1}, \ldots, h_{r}\right)
\end{array}
$$

where the right hand side is defined by the assumption of induction.

It is easy to see that the extension $\tilde{\psi}$ is well defined (i.e. it does not depend on the choice of presentation (3)). Next $\tilde{\psi}$ is continuous due to (2), and it is linear with respect to $h_{1}, \ldots, h_{r}$. Now Corollary 1.2.2 follows from a very general, simple, and well known lemma as follows.

1.2.3. Lemma. Let $X$ be a compact topological space. Let $F_{1}, \ldots, F_{t}$ be locally convex $\mathbb{C}$-linear topological spaces. Let

$$
\phi: X \times F_{1} \times \cdots \times F_{t} \rightarrow \mathbb{C}
$$

be a continuous map which is linear with respect to the last $t$ arguments.

Then there exist continuous semi-norms $\|\cdot\|_{1}, \ldots,\|\cdot\|_{t}$ on $F_{1}, \ldots, F_{t}$ respectively such that for any $x \in X, \xi_{1} \in F_{1}, \ldots, \xi_{t} \in F_{t}$ one has

$$
\left|\phi\left(x, \xi_{1}, \ldots, \xi_{t}\right)\right| \leq \prod_{i=1}^{t}\left\|\xi_{i}\right\|_{i} .
$$

Thus Corollary 1.2.2 is proved.

\subsection{Some valuation theory.}

1.3.1. Definition. a) A function $\phi: \mathcal{K}(V) \rightarrow \mathbb{C}$ is called a valuation if for any $K_{1}, K_{2} \in \mathcal{K}(V)$ such that their union is also convex one has

$$
\phi\left(K_{1} \cup K_{2}\right)=\phi\left(K_{1}\right)+\phi\left(K_{2}\right)-\phi\left(K_{1} \cap K_{2}\right) .
$$

b) A valuation $\phi$ is called continuous if it is continuous with respect to the Hausdorff metric on $\mathcal{K}(V)$. 
For classical theory of valuations we refer to the surveys of McMullen-Schneider [17] and McMullen [16]. For general background from convexity we refer to Schneider [18].

In [4] one has introduced a class $S V(V)$ of valuations called smooth valuations. We refer to [4] for an axiomatic definition. Here we only mention that $S V(V)$ is a $\mathbb{C}$-linear space (with the obvious operations) with a natural Fréchet topology. In this article we will need another description of $S V(V)$, given in Theorem 1.3.2 below.

Let us denote by ${ }^{\mathbf{C}} L$ the (complex) line bundle over $\mathbb{P}_{+}\left(V^{*}\right)$ whose fiber over $l \in \mathbb{P}_{+}\left(V^{*}\right)$ is equal to $l^{*} \otimes_{\mathbb{R}} \mathbb{C}$ (where $l^{*}$ denotes the dual space to $l$ ).

Note that for any convex compact set $A \in \mathcal{K}(V)$ the supporting functional $h_{A}$ is a continuous section of ${ }^{\mathrm{C}} L: h_{A} \in C\left(\mathbb{P}_{+}\left(V^{*}\right),{ }^{\mathrm{C}} L\right)$.

1.3.2. Theorem ([4], Corollary 3.1.7). There exists a continuous linear map

$$
\mathcal{T}: \bigoplus_{k=0}^{n} C^{\infty}\left(V \times \mathbb{P}_{+}\left(V^{*}\right)^{k},\left|\omega_{V}\right| \otimes^{C} L^{\otimes k}\right) \rightarrow S V(V)
$$

which is uniquely characterised by the following property: for any $k=0,1, \ldots, n$, any $\mu \in C^{\infty}\left(V,\left|\omega_{V}\right|\right)$, any strictly convex compact sets $A_{1}, \ldots, A_{k}$ with smooth boundaries, and any $K \in \mathcal{K}(V)$ one has

$$
\mathcal{T}\left(\mu \otimes h_{A_{1}} \otimes \cdots \otimes h_{A_{k}}\right)(K)=\left.\frac{\partial^{k}}{\partial \lambda_{1} \ldots \partial \lambda_{k}}\right|_{0} \mu\left(K+\sum_{i=1}^{k} \lambda_{i} A_{i}\right)
$$

where $\lambda_{i} \geq 0$ in the right hand side.

Moreover the map $\mathcal{T}$ is an epimorphism.

In [5] one has introduced for any smooth manifold $X$ a class of finitely additive measures on the family of simple subpolyhedra $\mathcal{P}(X)$. This class is denoted by $V^{\infty}(X)$. It is a $\mathbb{C}$-linear space (with the obvious operations). Then $V^{\infty}(X)$ has a natural Fréchet topology. Moreover in the case of a linear space $V$, the restriction of any element $\phi \in V^{\infty}(V)$ to $\mathcal{K}(V) \cap \mathcal{P}(V)$ has a (unique) extension by continuity in the Hausdorff metric to $\mathcal{K}(V)$, and this extension belongs to $S V(V)$. Thus one gets a linear map

$$
V^{\infty}(V) \rightarrow S V(V)
$$

In [5], Proposition 2.4.10, the following result was proved.

1.3.3. Proposition. The map $V^{\infty}(V) \rightarrow S V(V)$ constructed above is an isomorphism of Fréchet spaces.

\section{VAluations AND NORMAL CYCLES}

2.1. Normal cycles for convex sets. Let $V$ be a real vector space with finite dimension $n$. Let $K \in \mathcal{K}(V)$. Let $x \in K$.

2.1.1. Definition. The tangent cone to $K$ at $x$ is the closure of the set $\{y \in V \mid \exists \varepsilon>$ $0 x+\varepsilon y \in K\}$. We denote it by $T_{x} K$.

It is easy to see that $T_{x} K$ is a closed convex cone.

2.1.2. Definition. The conormal cone to $K$ at $x$ is the set

$$
\operatorname{Nor}^{*}(K, x):=\left\{y \in V^{*} \mid y(x) \leq 0 \forall x \in T_{x} K\right\} \text {. }
$$


Thus $\operatorname{Nor}^{*}(K, x)$ is also a closed convex cone. Also put

$$
\operatorname{Nor}^{*}(A):=\bigcup_{x \in K}\left(\{x\} \times \operatorname{Nor}^{*}(K, x)\right) .
$$

Fixing a euclidean metric (, ) on $V$, it will be convenient to define $\operatorname{Nor}(A) \subset V \times V$ as the image of $\operatorname{Nor}^{*}(A)$ under the induced identification $V \times V^{*} \simeq V \times V$. Finally, we put

$$
\operatorname{Nor}_{1}(K):=\{(x, v) \in \operatorname{Nor}(K):|v|=1\} .
$$

It is easy to see that $\operatorname{Nor}^{*}(K)$ (resp. $\left.\operatorname{Nor}(K)\right)$ is a closed $n$-dimensional subset of $T^{*} V=V \times V^{*}$ (resp. $T V$ ) invariant with respect to multiplication by non-negative numbers acting on the second factor.

Observe that $\operatorname{Nor}(K)$, and hence $\operatorname{Nor}^{*}(A)$ as well, is biLipschitz homeomorphic to $V$ : putting $p_{A}: V \rightarrow A$ for the nearest point projection, $V$ maps onto $\operatorname{Nor}(A)$ via the map $P_{A}: x \mapsto\left(p_{A}(x), x-p_{A}(x)\right)$, with inverse induced by $(x, y) \mapsto x+y$. It is clear that $P_{A}: V \rightarrow V \times V$ is a proper map.

It is useful to think of these objects as defining integral currents in the tangent and cotangent bundles of $V$. Given a smooth manifold $M$, put $\Omega_{c}^{k}(M)$ for the space of all compactly supported $C^{\infty}$ differential forms of degree $k$ on $M$. We recall ([9]) that the space $\mathbb{I}_{k}(M)$ of integral currents of dimension $k$ on $M$ is the space of all continuous linear functionals $T: \Omega_{c}^{k}(M) \rightarrow \mathbb{R}$ with the properties

- There is a sequence of bounded measurable subsets $E_{1}, E_{2}, \cdots \subset \mathbb{R}^{k}$ and locally Lipschitz maps $f_{i}: E_{i} \rightarrow M$ such that

$$
T=\sum_{i=1}^{\infty} f_{i *} \llbracket E_{i} \rrbracket,
$$

where $\llbracket E \rrbracket$ denotes the operation of integration of a $k$-form over $E$. Note that by Rademacher's theorem the derivative of $f$ exists almost everywhere, and constitutes a bounded measurable function. Thus the pull-backs $f_{i}^{*} \phi$ are integrable over the $E_{i}$, so the pushed forward currents $f_{i *} \llbracket E_{i} \rrbracket$, given by

$$
f_{i *} \llbracket E_{i} \rrbracket(\phi):=\int_{E_{i}} f_{i}^{*} \phi,
$$

are well-defined.

- For each compact set $C \subset M$ we have $\operatorname{mass}_{C}(T)<\infty$, where

$$
\operatorname{mass}_{C}(T):=\sup \left\{T(\phi): \operatorname{supp} \phi \subset C,\|\phi\|_{0} \leq 1\right\} .
$$

Here $\|\cdot\|_{0}$ denotes the $C^{0}$-norm.

- For each compact $C \subset M$,

$$
\operatorname{mass}_{C}(\partial T)<\infty
$$

Here $\partial T$ is the current of dimension $k-1$ given by $\partial T(\psi):=T(d \psi)$.

Equipping $M$ with a smooth Riemannian metric, the coflat seminorm $\|\phi\|^{b}$ of a form $\phi \in \Omega^{k}(M)$ is given by

$$
\|\phi\|^{b}:=\max \left\{\|\phi\|_{0},\|d \phi\|_{0}\right\} .
$$

Given $C \subset M$, we put

$$
\|T\|_{b}^{C}:=\sup \left\{|T(\phi)|:\|\phi\|^{b} \leq 1, \operatorname{supp} \phi \subset C\right\} .
$$


This is the restriction to the lattice $\mathbb{I}_{k}(M)$ of the flat seminorm relative to $C$. In the case $C=M$ we will omit the superscript. The local flat topology on $\mathbb{I}_{k}(M)$ is determined by the condition that $T_{1}, T_{2}, \cdots \rightarrow T$ iff

$$
\left\|T_{i}-T\right\|_{b}^{C} \rightarrow 0
$$

for every compact $C \subset M$.

Remark. Related to the flat seminorms is the integral flat metric

$$
\mathcal{F}(T):=\inf \left\{\operatorname{mass}_{M}(R)+\operatorname{mass}_{M}(S): R \in \mathbb{I}_{k}(M), S \in \mathbb{I}_{k+1}(M), T=R+\partial S\right\} .
$$

Clearly

$$
\|T\|_{b}^{M} \leq \mathcal{F}(T) .
$$

We now fix an orientation of $V$ and define the conic normal cycle of $A$ to be the integral current

$$
\vec{N}(A):=P_{A *} \llbracket V \rrbracket \in \mathbb{I}_{n}(V \times V),
$$

where $\llbracket V \rrbracket$ denotes the fundamental class of $V$. By [9], 4.1.14 and 4.1.24, the image of an integral current under a proper Lipschitz map is well-defined, and belongs to the class of integral currents. Note that $\partial \vec{N}(A)=\partial P_{A *} \llbracket V \rrbracket=P_{A *} \partial \llbracket V \rrbracket=0$. Likewise we define the conic conormal cycle $\vec{N}^{*}(A) \in \mathbb{I}_{n}\left(V \times V^{*}\right)$ as the image of $\vec{N}(A)$ under the identification $V \times V \rightarrow V \times V^{*}=T^{*} V$ arising from the euclidean structure. (Note that the image of $\vec{N}^{*}(A)$ under the antipodal map on the $V^{*}$ factor is identical to the characteristic cycle of $A$.) It is easy to see that the supports of $\vec{N}(A), \vec{N}^{*}(A)$ are $\operatorname{Nor}(A)$, $\operatorname{Nor}^{*}(A)$ respectively, and that $\operatorname{Nor}^{*}(A)$ and $\vec{N}^{*}(A)$ are independent of the choice of Euclidean metric.

Recall that $\mathcal{K}(V)$ denotes the metric space of all compact convex subsets of $V$, endowed with the Hausdorff metric. We endow $\mathbb{I}_{n}\left(V \times V^{*}\right)$ with the topology of local flat convergence.

2.1.3. Lemma. $\vec{N}^{*}$ is continuous as a map $\mathcal{K}(V) \rightarrow \mathbb{I}_{n}\left(V \times V^{*}\right)$.

Proof. Let $A_{0}, A_{1}, \cdots \in \mathcal{K}(V)$, with $A_{i} \rightarrow A_{0}$. Clearly the nearest point projections $p_{A_{i}}$ converge uniformly to $p_{A_{0}}$, hence $P_{A_{i}} \rightarrow P_{A_{0}}$ uniformly as well. It now follows from the discussion in 4.1.14 of [9], and the definition of $\vec{N}^{*}$, that $\vec{N}^{*}\left(A_{i}\right) \rightarrow \vec{N}^{*}\left(A_{0}\right)$ in the local flat topology.

It is convenient to introduce a different characterization of $\vec{N}^{*}(A)$. We say that a current in $\mathbb{I}_{n}\left(V \times V^{*}\right)$ is Lagrangian if it annihilates the ideal of all multiples of the canonical symplectic form $\omega$. The terminology is motivated by the obvious fact that if $M \subset V \times V^{*}$ is a smooth oriented $n$-dimensional submanifold, then the current given by integration of $n$-forms over $M$ is Lagrangian iff $M$ is a Lagrangian submanifold of $V \times V^{*}$ in the usual sense.

2.1.4. Lemma. If $A \in \mathcal{K}(V)$, then $\vec{N}^{*}(A)$ is a Lagrangian current.

Proof. If $A$ has smooth boundary and non-empty interior, then $\vec{N}^{*}(A)$ decomposes as the sum of two terms. The first is integration over the image of $A$ itself under the zero map $V \rightarrow V^{*}$. This current is obviously Lagrangian. The second is integration over the bundle of outward conormal rays to the smooth hypersurface $\partial A$. The conormal bundle of a submanifold is a classical example of a Lagrangian submanifold, hence the second term is Lagrangian as well. 
As is well known, every element $A \in \mathcal{K}(V)$ may be approximated in the Hausdorff metric by a sequence $A_{1}, A_{2}, \ldots$ of smooth bodies with non-empty interior. By Lemma 2.1 .3 , the conic conormal cycles $\vec{N}^{*}\left(A_{i}\right)$ converge in the locally flat topology to $\vec{N}^{*}(A)$. But locally flat convergence implies weak convergence, so the latter current must annihilate the symplectic ideal since the the former do.

We now recall the main theorem of [10].

2.1.5. Theorem. Let $W$ be an oriented real vector space of dimension $n$. If $f$ : $W \rightarrow \mathbb{R}$ is locally Lipschitz, then there is at most one closed Lagrangian integral current $T \in \mathbb{I}_{n}\left(W \times W^{*}\right)$ such that

- $T$ is locally vertically bounded, i.e. supp $T \cap\left(C \times W^{*}\right)$ is compact for every compact $C \subset W$; and

- if $\phi: W \times W^{*} \rightarrow \mathbb{R}$ is a smooth compactly supported function and $d \operatorname{vol}_{W}$ is a (positive) volume form for $W$, then

$$
T\left(\phi \pi_{W}^{*} d \operatorname{vol}_{W}\right)=\int_{W} \phi(x, d f(x)) d \operatorname{vol}_{W}
$$

where $\pi_{W}: W \times W^{*} \rightarrow W$ is the canonical projection.

If $f$ is convex, then this $T$ exists.

Here $\pi_{W}: W \times W^{*} \rightarrow W$ denotes the projection to the first factor. We will call this current $T$ the differential cycle of $f$, denoted here by $D(f)$.

Remarks. 1. The differential $d f(x)$ exists for almost every $x$ by Rademacher's theorem. The resulting map $d f$ is measurable, so (5) makes sense.

2. The point is that if $f$ is smooth, then the current $D(f)$ is simply integration over the graph of the differential $d f$. If $f$ is convex, then the graph of the subgradient of $f$ is a Lipschitz submanifold of $W \times W^{*}$ and inherits a natural orientation from that of $W$; the differential current $D(f)$ is then given by integration over the graph of the subgradient.

3. In fact a stronger form of the theorem is true: the condition that $f$ be locally Lipschitz may be replaced by the statement $f \in W_{l o c}^{1,1}$ (i.e. $d f \in L_{l o c}^{1}$ ); and the first condition on $T$ may be replaced by the requirement that the restriction $T\left\llcorner\left(C \times W^{*}\right)\right.$ have finite mass for every compact $C \subset W$.

Sketch of proof. It is enough to show that if $T$ satisfies the first condition, and additionally annihilates all functional multiples of $\pi_{W}^{*} d \operatorname{vol}_{W}$, then $T=0$. The proof of this statement is modeled on a well-known fact about smooth Lagrangian submanifolds $L \subset T^{*} W$ with the property that $\pi_{W} \mid L$ is a submersion: locally, such a submanifold is a fiber bundle over its image $\Lambda \subset W$, with fibers of the form $d g(x)+\nu_{x}^{*} \Lambda$, where $g$ is a smooth function and $\nu_{x}^{*} \Lambda$ is the conormal fiber to $\Lambda$ at $x$ (cf. [14]). In particular, the fibers are unbounded. A weak form of this description applies to a rectifiable Lagrangian carrier of $L$ on the set of points where the projection to $W$ has maximal rank.

Given $A \in \mathcal{K}(V)$, we denote by $h_{A}: V^{*} \rightarrow \mathbb{R}$ the support function of $A$ given by

$$
h_{A}(\lambda):=\sup _{x \in A} \lambda(x) .
$$

It is well known, and easy to prove, that $h_{A}$ is sublinear, i.e. convex and positively homogeneous of degree 1 [18]. In particular it is Lipschitz, hence differentiable for 
a.e. $y \in V^{*}$ by Rademacher's theorem. In this case the differential $d h_{A}(\lambda) \in V$ has a particular geometric meaning:

2.1.6. Proposition. If $h_{A}$ is differentiable at $\lambda \in V^{*}$, then $x:=d h_{A}(\lambda) \in V$ is the unique point in $A$ at which $\lambda$ supports $A$, i.e. such that $\lambda(A) \subset(-\infty, \lambda(x)]$.

Proof. [18], Corollary 1.7.3.

2.1.7. Proposition. Let $i: V \times V^{*} \rightarrow V^{*} \times V$ denote the interchange map $i(x, y)=$ $(y, x)$. If $A \in \mathcal{K}(V)$, then

$$
i_{*} \vec{N}^{*}(A)=D\left(h_{A}\right)
$$

Proof. It is enough to check that $i_{*} \vec{N}^{*}(A)$ satisfies the conditions of Theorem 2.1.5 for $f=h_{A}$. The first is trivial: since $\vec{N}^{*}(A)$ is supported in $A \times V^{*}$, it is clear that $i_{*} \vec{N}^{*}(A)$ is supported in $V^{*} \times A$ and hence is even globally vertically bounded.

To prove the second, we pass to the dual setting using our fixed euclidean structure $(\cdot, \cdot)$ on $V$. Abusing notation, we again denote by $h_{A}: V \rightarrow \mathbb{R}$ the support function

$$
h_{A}(x):=\sup _{y \in A}(x, y) .
$$

Put $q_{A}(y)=y-p_{A}(y)$, and let $p_{1}, p_{2}: V \times V \rightarrow V$ be the projections to the first and second factors, respectively. We must show that for smooth compactly supported functions $\varphi: V \times V \rightarrow \mathbb{R}$,

$$
i_{*} \vec{N}(A)\left(\varphi p_{1}^{*} \mathrm{~d} v o l_{V}\right)=\int_{V} \varphi\left(x, \nabla h_{A}(x)\right) d x
$$

Recalling (4), the left-hand side may be expressed as

$$
\begin{aligned}
i_{*} P_{A *}[V]\left(\varphi p_{1}^{*} \mathrm{~d} \mathrm{vol}_{V}\right) & =\int_{V} P_{A}^{*} i^{*}\left(\varphi p_{1}^{*} \mathrm{~d} \mathrm{vol}_{V}\right) \\
& =\int_{V} \varphi\left(y-p_{A}(y), p_{A}(y)\right) P_{A}^{*} p_{2}^{*} \mathrm{~d} \operatorname{vol}_{V}(y) \\
& =\int_{V} \varphi\left(q_{A}(y), p_{A}(y)\right) q_{A}^{*} \operatorname{d~vol}_{V}(y) \\
& =\int_{V} \varphi\left(q_{A}(y), \nabla h_{A}\left(q_{A}(y)\right)\right) q_{A}^{*} \operatorname{dvol}_{V}(y),
\end{aligned}
$$

by Proposition 2.1.6. Since $q_{A}^{-1}(y)$ is a singleton for a.e. $y \in V$, the desired relation (8) follows from the change of variables formula.

In fact the inverse map to $\vec{N}^{*}$ is also well-defined and continuous:

2.1.8. Corollary. If $A, B \in \mathcal{K}(V)$ and $\vec{N}^{*}(A)=\vec{N}^{*}(B)$, then $A=B$. If $A_{0}, A_{1}, A_{2}$, $\cdots \in \mathcal{K}(V)$ and $\vec{N}^{*}\left(A_{i}\right) \rightarrow \vec{N}^{*}\left(A_{0}\right)$ in the local flat topology, then $A_{i} \rightarrow A_{0}$ in the Hausdorff metric topology. 
Proof. If $\vec{N}^{*}(A)=\vec{N}^{*}(B)$, then $D\left(h_{A}\right)=D\left(h_{B}\right)$ by Proposition 2.1.7. It follows at once that $d h_{A}=d h_{B}$ a.e. in $V^{*}$. Since a Lipschitz function on a euclidean space with derivative a.e. equal to zero is constant, and $h_{A}(0)=h_{B}(0)=0$, we conclude that $h_{A}=h_{B}$. Therefore $A=B$.

To prove the second statement, note first that all of the $A_{i}$ must lie within a sufficiently large fixed compact subset of $V$ : for there exists a smooth differential form $\kappa_{1}$ on $T^{*} V$ such that $\vec{N}^{*}(A)\left(\kappa_{1}\right)$ is the mean breadth of $A$, for all $A \in \mathcal{K}(V)$ (cf. [11]). Thus the mean breadth of the $A_{i}$ converges to that of $A_{0}$. But the mean breadth of a convex body dominates its diameter, so we conclude that the diameters of the $A_{i}$ are uniformly bounded. Furthermore there exists another smooth differential form $\kappa_{0}$ such that if $\phi: V \rightarrow \mathbb{R}$ is a smooth compactly supported function whose restriction to $A_{0}$ is equal to 1 , then $\vec{N}^{*}\left(A_{0}\right)\left(\pi^{*} \phi \kappa_{0}\right)=1$ where $\pi: T^{*} V \rightarrow V$ is the canonical projection. Thus $\vec{N}^{*}\left(A_{i}\right)\left(\pi^{*} \phi \kappa_{0}\right) \neq 0$ for all sufficiently large $i$, and in particular $A_{i}=\pi\left(\operatorname{supp} \vec{N}^{*}\left(A_{i}\right)\right)$ has a non-empty intersection with supp $\phi$ for such $i$.

Thus the Blaschke Selection Theorem implies that there exists a subsequence $A_{i^{\prime}}$ converging in the Hausdorff metric to some $B_{0} \in \mathcal{K}(V)$. By Lemma 2.1.3, $\vec{N}^{*}\left(A_{0}\right)=\lim _{i^{\prime} \rightarrow \infty} \vec{N}^{*}\left(A_{i^{\prime}}\right)=\vec{N}^{*}\left(B_{0}\right)$; by the assertion above, $A_{0}=B_{0}$. Since this outcome is independent of the chosen convergent subsequence it follows that the entire sequence of the $A_{i}$ converges to $A_{0}$.

2.1.9. Proposition. If $f, g, \min (f, g): W \rightarrow \mathbb{R}$ are convex, then

$$
D(\max (f, g))+D(\min (f, g))=D(f)+D(g) .
$$

Proof. It is enough to show that $D(f)+D(g)-D(\min (f, g))$ satisfies the conditions of Theorem 2.1.5, with $f$ replaced by the function $\max (f, g)$. All of them are immediate except for the last one, and for this it is enough to show that

$$
\{d(\max (f, g))(x), d(\min (f, g))(x)\}=\{d f(x), d g(x)\}
$$

for a.e. $x \in W$ at which all four differentials exist (which happens a.e. in $W$ by Rademacher's theorem).

This is obvious when $f(x) \neq g(x)$. On the other hand, let $E$ denote the set of points $x$ such that $f(x)=g(x)$ and both of $f, g$ are differentiable at $x$. By classical measure theory, $E$ has density 1 at a.e. point $x \in E$. If $x$ is such a point, then clearly $d f(x)=d g(x)$. Therefore this common value is also equal to both $d \max (f, g)(x)$ and $d \min (f, g)(x)$.

2.1.10. Corollary. If $A, B, A \cup B \in \mathcal{K}(V)$, then

$$
\vec{N}^{*}(A \cup B)+\vec{N}^{*}(A \cap B)=\vec{N}^{*}(A)+\vec{N}^{*}(B) .
$$

Proof. If $A \cup B \in \mathcal{K}(V)$, then $h_{A \cup B}=\max \left(h_{A}, h_{B}\right)$ and $h_{A \cap B}=\min \left(h_{A}, h_{B}\right)$. Therefore (13) follows at once from Propositions 2.1.7 and 2.1.9.

It is sometimes convenient to instead consider the (non-conic) normal cycle $N(A)$ in the tangent sphere bundle $V \times S(V)$, and the corresponding conormal cycle $N^{*}(A)$ in the cotangent ray bundle $V \times \mathbb{P}_{+}\left(V^{*}\right)$. To define $N(A)$, let $r: V \rightarrow$ $[0, \infty)$ denote the length function induced by the fixed euclidean metric. Then

$$
N(A):=g_{*}\left(\left\langle\vec{N}(A), r \circ p_{2}, 1\right\rangle\right) \in \mathbb{I}_{n-1}(V \times S(V)),
$$


where $\langle T, f, c\rangle$ denotes the slice of the current $T$ by the function $f$ at the value $c$ (cf. [9], 4.3) and $g: V \times(V-\{0\}) \rightarrow V \times S(V)$ is the normalizing map $(x, y) \mapsto$ $\left(x, \frac{y}{\mid y}\right)$. Note that the slicing operation is well-defined for a.e. $c$ whenever $T$ is an integral current and $f$ is a Lipschitz function whose restriction to the support of $T$ is proper, and may be thought of as the intersection of $T$ with the level set $f^{-1}(c)$. In the present case the slice is well-defined at every value of $r$ since, putting $\theta_{c}(x, y):=(x, c y)$ for $c>0$,

$$
\langle\vec{N}(A), r, a\rangle=\theta_{c *}\left\langle\vec{N}(A), r, \frac{a}{c}\right\rangle
$$

— this in view of the facts

$$
\begin{aligned}
\theta_{c *} \vec{N}(A) & =\vec{N}(A), \\
r \circ \theta_{c} & =c r
\end{aligned}
$$

and the general formula

$$
\left\langle h_{*} T, f, c\right\rangle=h_{*}\langle T, f \circ h, c\rangle
$$

(cf. [9], 4.3.2(7)).

The conormal cycle $N^{*}(A)$ is then the image of $N(A)$ under the natural map $V \times S(V) \rightarrow V \times \mathbb{P}_{+}\left(V^{*}\right)$ induced by the euclidean metric. It is clear that $N^{*}(A)$ does not depend on the choice of this metric. Recall that $V \times \mathbb{P}_{+}\left(V^{*}\right)$ carries a natural contact structure, and a choice of metric even determines a particular global contact 1-form. The current $N^{*}(A)$ is Legendrian in the sense that it annihilates every element of the ideal generated by any such 1-form.

The conic normal cycle may be reconstructed from the normal cycle in a canonical way. Given a manifold $M$, put $\mathbb{I}_{k}^{c}(M)$ for the space of all compactly supported integral currents of dimension $k$ on $M$, where the topology on this space is determined by the condition that $T_{i} \rightarrow T_{0} \in \mathbb{I}_{k}^{c}(M)$ iff $T_{i} \rightarrow T_{0}$ in the local flat topology and all of the $T_{i}$ are supported in a single compact set $C \subset M$. Define $f: V \times S(V) \times \mathbb{R} \rightarrow V \times V$ by $f(x, v ; t):=(x, t v)$. Also define $g: V \times \mathbb{R} \rightarrow V$ by $g(x, t):=t x$ and put $h: V \times S(V) \rightarrow V$ for the projection. Now define $\gamma: \mathbb{I}_{n-1}^{c}(V \times S(V)) \rightarrow \mathbb{I}_{n}(V \times V)$ by

$$
\gamma(T):=f_{*}(T \times[0, \infty))+g_{*}\left(h_{*} T \times \llbracket 0,1 \rrbracket\right) \times \llbracket 0 \rrbracket .
$$

Since $f$ is proper this map is continuous in view of the topology given above on $\mathbb{I}_{n-1}^{c}$. If $\partial T=0$, then the first factor in the second term above may be characterized as the unique compactly supported current in $V$ with boundary equal to $h_{*} T$.

2.1.11. Proposition. If $A \in \mathcal{K}(V)$, then $\gamma(N(A))=\vec{N}(A)$.

Proof. For $r \geq 0$, put $A_{r}:=\{x \in V: \operatorname{dist}(x, A)=r\}$. Then $N(A)=P_{A *} \llbracket A_{1} \rrbracket$ and the map $Q:(x, t) \mapsto t\left(x-p_{A}(x)\right)+p_{A}(x)$ is an orientation-preserving locally biLipschitz homeomorphism $A_{1} \times(0, \infty) \rightarrow V \backslash A$. These maps satisfy the relations

$$
\begin{aligned}
P_{A} \circ Q & =f \circ\left(P_{A} \times \mathrm{id}\right), \\
h \circ P_{A} & =p_{A} .
\end{aligned}
$$

Therefore

$$
h_{*} N(A)=p_{A *} \llbracket A_{1} \rrbracket=\partial \llbracket A \rrbracket,
$$


and, using the characterization above of the second term in (15),

$$
\begin{aligned}
\vec{N}(A) & :=P_{A *} \llbracket V \rrbracket \\
& =P_{A *} \llbracket A \rrbracket+P_{A *} \llbracket V \backslash A \rrbracket \\
& =\llbracket A \rrbracket \times \llbracket 0 \rrbracket+P_{A *} Q_{*} \llbracket A_{1} \times(0, \infty) \rrbracket \\
& =g_{*}(\partial \llbracket A \rrbracket \times \llbracket 0,1 \rrbracket) \times \llbracket 0 \rrbracket+f_{*}\left(P_{A *} \llbracket A_{1} \rrbracket \times \llbracket 0, \infty \rrbracket\right) \\
& =g_{*}\left(h_{*} N(A) \times \llbracket 0,1 \rrbracket\right) \times \llbracket 0 \rrbracket+f_{*}(N(A) \times \llbracket 0, \infty \rrbracket),
\end{aligned}
$$

as claimed.

It is immediate from the definition that the normal and conormal cycles share the basic properties of their conic counterparts described above. For brevity we give the explicit statements only in the conormal case:

2.1.12. Proposition. $N^{*}$ is a continuous injection $\mathcal{K}(V) \rightarrow \mathbb{I}_{n-1}\left(V \times \mathbb{P}_{+}\left(V^{*}\right)\right)$, and the inverse map (defined on the image) is continuous, where the topology on $\mathbb{I}_{n-1}\left(V \times \mathbb{P}_{+}\left(V^{*}\right)\right)$ is the local flat topology. $N^{*}$ is a valuation in the sense that if $A, B, A \cup B \in \mathcal{K}(V)$, then $N^{*}(A \cup B)+N^{*}(A \cap B)=N^{*}(A)+N^{*}(B)$.

Put $C_{\mathrm{b}}^{1}$ for the normed space of all $C^{1}$-smooth differential forms $\phi$ of degree $n-1$ on $\left(V \times \mathbb{P}_{+}\left(V^{*}\right)\right)$, with finite coflat norm. The preceding discussion yields the following.

2.1.13. Theorem. The map $\mathcal{K}(V) \times C_{\mathrm{b}}^{1} \rightarrow \mathbb{R}$ given by

$$
(A, \phi) \mapsto N^{*}(A)(\phi)
$$

is continuous.

Remark. The space $C_{b}^{1}$ may be replaced by the space of all flat cochains (cf. [20], p. 233) with finite coflat norm.

Proof. Suppose $A_{1}, A_{2}, \cdots \rightarrow A_{0}$ in $\mathcal{K}(V)$ and $\phi_{1}, \phi_{2}, \cdots \rightarrow \phi_{0}$ in $C_{b}^{1}$. By Proposition 2.1.12, given $\varepsilon>0$ there is $M \in \mathbb{N}$ such that

$$
\begin{array}{r}
\left.\| N^{*}\left(A_{i}\right)-N^{*}\left(A_{0}\right)\right) \|_{b}<\varepsilon, \\
\left\|\phi_{i}-\phi_{0}\right\|^{b}<\varepsilon
\end{array}
$$

for $i \geq N$. Thus

$$
\begin{aligned}
& \left|N^{*}\left(A_{i}\right)\left(\phi_{i}\right)-N^{*}\left(A_{0}\right)\left(\phi_{0}\right)\right| \\
& \quad \leq\left|N^{*}\left(A_{i}\right)\left(\phi_{i}\right)-N^{*}\left(A_{0}\right)\left(\phi_{i}\right)\right|+\left|N^{*}\left(A_{0}\right)\left(\phi_{i}\right)-N^{*}\left(A_{0}\right)\left(\phi_{0}\right)\right| \\
& \quad \leq\left\|N^{*}\left(A_{i}\right)-N^{*}\left(A_{0}\right)\right\|_{b}\left\|\phi_{i}\right\|^{b}+\operatorname{mass}\left(N^{*}\left(A_{0}\right)\right)\left\|\phi_{i}-\phi_{0}\right\|_{0} \\
& \quad<\varepsilon\left(\left\|\phi_{0}\right\|^{b}+\varepsilon+\operatorname{mass} N^{*}\left(A_{0}\right)\right),
\end{aligned}
$$

which proves the desired assertion.

2.2. Normal cycles for more general sets. Normal cycles are also available for a wide class of sets other than convex ones. First let us define it in the class of sets presentable as finite unions of convex compact sets. Let

$$
X=\bigcup_{i=1}^{N} A_{i}, A_{i} \in \mathcal{K}(V) .
$$


Set

$$
\begin{aligned}
N^{*}(X) & :=\sum_{I \subset\{1, \ldots, N\}, I \neq \emptyset}(-1)^{|I|+1} N^{*}\left(\bigcap_{i \in I} A_{i}\right), \\
\vec{N}^{*}(X) & :=\sum_{I \subset\{1, \ldots, N\}, I \neq \emptyset}(-1)^{|I|+1} \vec{N}^{*}\left(\bigcap_{i \in I} A_{i}\right) .
\end{aligned}
$$

Using Corollary 2.1.10 it is easy to check that the definitions of $N^{*}(X)$ and $\vec{N}^{*}(X)$ do not depend on a choice of presentation (16).

Let us define $N^{*}(X)$ and $\vec{N}^{*}(X)$ where $X$ is a compact smooth submanifold with boundary. For any point $x \in X$ let us define the tangent cone to $X$ at $x$, denoted by $T_{x} X$, as the set

$$
T_{x} X:=\left\{\xi \in T_{x} V \mid \text { there exists a } C^{1}-\text { map } \gamma:[0,1] \rightarrow X \text { such that } \gamma^{\prime}(0)=\xi\right\} .
$$

It is easy to see that $T_{x} X$ coincides with the usual tangent space if $x$ is an interior point of $X$, and it is a halfspace if $x$ belongs to the boundary of $X$. Define

$$
\operatorname{Nor}^{*}(X):=\bigcup_{x \in X}-\left(T_{x} X\right)^{o}
$$

where for a convex cone $C$ in a linear space $W$ one denotes $C^{o}$ its dual cone in $W^{*}$ :

$$
C^{o}:=\left\{y \in W^{*} \mid y(x) \geq 0 \text { for any } x \in C\right\} .
$$

Clearly $\operatorname{Nor}^{*}(X)$ is invariant under the group $\mathbb{R}_{>0}$ of positive real numbers acting on the cotangent bundle $T^{*} V$ by multiplication along the fibers. The sets $\operatorname{Nor}(X)$ and $\operatorname{Nor}_{1}(X)$ are now defined as in Definition 2.1.2.

The corresponding current $N^{*}(X)$ is more naturally constructed in the more general context of semi-convex sets $X$ (cf. [21], [8]). Put $p_{X}: X_{[0, r)} \rightarrow X$ for the nearest point projection to $X$, defined for the set $X_{[0, r)}:=\{x \in V: \operatorname{dist}(x, X)<r\}$, where $r:=\operatorname{reach}(X)$ of $X$, and $P_{X}(x):=\left(p_{X}(x), x-p_{X}(x)\right)$. Put

$$
\vec{N}^{\prime}(X):=P_{X *} \llbracket X_{[0, r)} \rrbracket,
$$

where the domain $X_{[0, r)}$ inherits its orientation from $V$. Choose a diffeomorphism $f:[0, r) \rightarrow[0, \infty)$, and put $F(x, v):=\left(x, f(|v|) \frac{v}{|v|}\right)$. Now put

$$
\vec{N}(X):=F_{*} \vec{N}^{\prime}(X) .
$$

From this current we may construct the currents $\vec{N}^{*}(X), N(X), N^{*}(X)$ as in the remarks surrounding (14). It is easy to see that if $X$ is convex, then these definitions of $N^{*}(X)$ and $\vec{N}^{*}(X)$ coincide with the previous ones. Furthermore supp $N^{*}(X)=$ $\operatorname{Nor}^{*}(X)$ and supp $N(X)=\operatorname{Nor}_{1}(X)$.

The normal cycle of subanalytic subsets was defined in [13] using tools from geometric measure theory (in fact Theorem 3.2 of [13] gives a unique characterization of the normal cycle of an arbitrary compact set in $\mathbb{R}^{n}$, dual to Theorem 2.1.5). A similar notion of characteristic cycle of subanalytic subsets was introduced independently in [15] using tools from sheaf theory.

Conormal cycles transform in a natural way under diffeomorphisms. We will only need this fact in the smooth case:

2.2.1. Lemma. Let $X \subset V$ be a compact domain with smooth boundary, and $U \supset X$ an open neighborhood. Let $f: U \rightarrow W \subset V$ be an orientation-preserving diffeo- 
morphism, and let $\bar{f}: U \times \mathbb{P}_{+}\left(V^{*}\right) \rightarrow W \times \mathbb{P}_{+}\left(V^{*}\right)$ be the natural lift of $f$ defined by

$$
\bar{f}(x,[\lambda]):=\left(f(x),\left[\left(f^{-1}\right)^{*} \lambda\right]\right) .
$$

Then

$$
\bar{f}_{*} N^{*}(X)=N^{*}(f(X)) .
$$

Proof. It is clear that $\bar{f}$ maps the manifold of outward conormal lines to $\partial X$ diffeomorphically onto that of $f(\partial X)=\partial f(X)$. The cycles $N^{*}(X)$ and $N^{*}(f(X))$ are the fundamental classes of these manifolds, and therefore $\bar{f}_{*} N^{*}(X)= \pm N^{*}(f(X))$. To see that the sign is positive, we note that the orientations of the fundamental classes are determined by the relations

$$
p_{1 *} N^{*}(X)=\partial \llbracket X \rrbracket, p_{1 *} N^{*}(f(X))=\partial \llbracket f(X) \rrbracket .
$$

Since $f$ preserves orientation by hypothesis,

$$
p_{1 *} \bar{f}_{*} N^{*}(X)=f_{*} \partial \llbracket X \rrbracket=\partial \llbracket f(X) \rrbracket=p_{1 *} N^{*}(f(X)),
$$

which establishes the claim.

M. Zähle has proved the following fundamental approximation theorem:

2.2.2. Theorem. Let $X \subset V$ be a compact domain with smooth boundary. There exists a sequence of polyhedra $P_{1}, P_{2}, \cdots \subset V$ such that

$$
\lim _{i \rightarrow \infty} N\left(P_{i}\right)=N(X) .
$$

Proof. This is [21], Theorem 1. The proof given there may be simplified as follows.

One may show, along the lines of [21] or [12], that there exists a sequence of polyhedra $P_{i}$ and a constant $C<\infty$ such that

$$
\begin{aligned}
\operatorname{mass}\left(N\left(P_{i}\right)\right) & \leq C, \\
\operatorname{supp} N\left(P_{i}\right) & \rightarrow \operatorname{supp} N(X), \\
\llbracket P_{i} \rrbracket & \rightarrow \llbracket X \rrbracket .
\end{aligned}
$$

Clearly the relation $(20)$ implies that $\partial \llbracket P_{i} \rrbracket \rightarrow \partial \llbracket X \rrbracket$.

By the compactness theorem for integral currents $([19])$ and the constancy theorem ([9], p. 357), the relations (18) and (19) imply that there is a subsequence $N\left(P_{i^{\prime}}\right) \rightarrow k N(X)$ for some integer $k$. Thus $\partial \llbracket P_{i^{\prime}} \rrbracket=\pi_{*} N\left(P_{i^{\prime}}\right) \rightarrow k \pi_{*} N(X)=$ $k \partial \llbracket X \rrbracket$, so $(20)$ implies that $k=1$. Since this is independent of the subsequence chosen, the result follows.

2.2.3. Corollary. In the scenario of Theorem 2.2.2 we also have

$$
\lim _{i \rightarrow \infty} \vec{N}\left(P_{i}\right)=\vec{N}(X) .
$$

Proof. This follows at once from Theorem 2.2.2 and Proposition 2.1.11 since the map $\gamma$ occurring there is continuous. 


\section{Auxiliary Results}

The goal of this section is to prove some technical results which will be used in the construction of the product on valuations in Section 4. The main results of this section are Lemmas 3.1.10, 3.1.12, and Proposition 3.1.13.

Let $V$ be an $n$-dimensional real vector space. As usual we fix a Euclidean metric on $V$. In this section we will also fix a compact smooth $n$-dimensional submanifold with boundary $X^{\prime} \subset V \times V$ which projects diffeomorphically onto its images in $V$ under both projections $p_{1}, p_{2}: V \times V \rightarrow V$. We also fix a compact submanifold with boundary $X \subset X^{\prime}$ such that $X \cap \partial X^{\prime}=\emptyset$. We will denote throughout this section

$$
\tilde{p}_{1}, \tilde{p}_{2}: X^{\prime} \rightarrow V
$$

as the restrictions of the projections $p_{1}, p_{2}$ respectively to $X^{\prime}$.

For a domain $\Omega \subset V$ with smooth boundary let us denote by $k_{1}(\Omega, s), \ldots$, $k_{n-1}(\Omega, s)$ the principal curvatures at a point $s \in \partial \Omega$.

3.1.1. Lemma. Let $A_{1}, \ldots, A_{k} \subset V$ be compact strictly convex subsets with smooth boundaries. Then there exists a constant $C>0$ (depending on these subsets) such that for any $\lambda_{1}, \ldots, \lambda_{k} \geq 0$ with $\sum_{i=1}^{k} \lambda_{i}=1$ one has for any $1 \leq l \leq n-1$

$$
\frac{1}{C} \leq k_{l}\left(\sum_{i=1}^{k} \lambda_{i} A_{i}, s\right) \leq C
$$

for any $s \in \partial\left(\sum_{i=1}^{k} \lambda_{i} A_{i}\right)$.

Proof. For convenience let us fix on $V$ an orthonormal coordinate system $\left(x_{1}, \ldots\right.$, $\left.x_{n}\right)$. The principal curvatures $k_{l}(A)$ of a strictly convex compact set $A$ can be estimated from both sides via the eigenvalues of the Hessian of the supporting functional $\operatorname{Hess}\left(h_{A}\right):=\left(\frac{\partial^{2} h_{A}}{\partial x_{i} \partial x_{j}}\right)$ restricted to the tangent bundle to the unit sphere $T S^{n-1}$. Let us denote that $H_{i}:=\left.\operatorname{Hess}\left(h_{A_{i}}\right)\right|_{T S^{n-1}}, i=1, \ldots, k$.

Let us denote by $c^{\prime}$ and $C^{\prime}$ the minimum and the maximum respectively over the unit sphere $S^{n-1}$ of all the eigenvalues of all $H_{i}$ 's. Then clearly $0<c^{\prime}<C^{\prime}<\infty$. Then

$$
\left(\sum_{i} \lambda_{i}\right) c^{\prime} I d_{n-1} \leq \sum_{i} \lambda_{i} H_{i} \leq\left(\sum_{i} \lambda_{i}\right) C^{\prime} \cdot I d_{n-1} .
$$

The lemma follows.

3.1.2. Proposition. Let $\delta_{0}, \delta_{1}>0$ be given. Suppose $X \subset V$ is a compact subset with reach $(X)>\delta_{0}$. Suppose also that $A \subset V$ is a strictly convex compact set with smooth boundary, containing the origin in the interior, and such that all principal curvatures $k_{l}$ of $\partial A$ satisfy $k_{l} \geq \delta_{1}$. Then for any $0<\varepsilon \leq \delta_{0} \delta_{1}$, the map

$$
\xi_{A, \varepsilon}: V \times \mathbb{P}_{+}\left(V^{*}\right) \times[0,1] \rightarrow V
$$

given by

$$
\xi_{A, \varepsilon}(p, n, t)=p+\varepsilon \cdot t \nabla h_{A}(n)
$$

is a homeomorphism of $N(X) \times(0,1]$ onto $(X+\varepsilon A) \backslash X$.

For the proof we will need the two assertions, both known, of the following lemma. 
3.1.3. Lemma.

- If $\operatorname{reach}(X)>\delta_{0}$ and $n_{i} \in \operatorname{Nor}\left(X, x_{i}\right), i=0,1$, with $\left|n_{0}\right|=$ $\left|n_{1}\right|=1$, then

$$
\left(x_{1}-x_{0}, n_{1}-n_{0}\right) \geq-\delta_{0}^{-1}\left|x_{1}-x_{0}\right|^{2} .
$$

- Let $A$ be a convex body with smooth boundary, with all principal curvatures $k_{l} \geq \delta_{1}$. Suppose $x_{i} \in \partial A$, with outward normals $n_{i}, i=0,1$. Then

$$
\left(x_{1}-x_{0}, n_{1}-n_{0}\right) \geq \delta_{1}\left|x_{1}-x_{0}\right|^{2} .
$$

Proof. The first assertion follows at once from [8], Theorem 4.8 (7).

The second assertion may be deduced as follows. It is easy to see using Schur's theorem ([7]) that if $B$ is a ball of radius $\delta_{1}^{-1}$ passing through $x_{0}$, and with outward normal $n_{0}$ there, then $B \supset A$. On the other hand an elementary calculation shows that $\left(n_{0}, x_{0}-p\right) \geq \frac{\delta_{1}}{2}\left|p-x_{0}\right|^{2}$ for every $p \in B$. In particular $\left(n_{0}, x_{0}-x_{1}\right) \geq$ $\frac{\delta_{1}}{2}\left|x_{1}-x_{0}\right|^{2}$. Adding this to the analogous inequality for $x_{1}$ gives $(22)$.

Proof of Proposition 3.1.2. The proposition is equivalent to the assertion that if $r \leq \delta_{0} \delta_{1}$, then there is no translate $p-r A$ of $r(-A)$ with interior disjoint from $X$ and intersecting $X$ in two distinct points.

Suppose there is such a translate, with $x_{0}, x_{1} \in X \cap(p-r A), x_{0} \neq x_{1}$. Let $a_{i}:=r^{-1}\left(p-x_{i}\right) \in \partial A$, and let $n_{i}$ be the outward unit normals to $A$ at $a_{i}, i=0,1$. By $(22)$,

$$
r^{-1}\left(x_{0}-x_{1}, n_{1}-n_{0}\right)=\left(a_{1}-a_{0}, n_{1}-n_{0}\right) \geq \delta_{1}\left|a_{1}-a_{0}\right|^{2}=\delta_{1} r^{-2}\left|x_{1}-x_{0}\right|^{2}
$$

or

$$
\left(x_{0}-x_{1}, n_{1}-n_{0}\right) \geq r^{-1} \delta_{1}\left|x_{1}-x_{0}\right|^{2} .
$$

On the other hand it is clear that $n_{i} \in \operatorname{Nor}\left(X, x_{i}\right)$, so (21) gives

$$
\left(x_{0}-x_{1}, n_{1}-n_{0}\right) \leq \delta_{0}^{-1}\left|x_{0}-x_{1}\right|^{2} .
$$

Thus $r \geq \delta_{0} \delta_{1}$, as claimed.

Recall that we denote by $L$ the (real) line bundle over $\mathbb{P}_{+}\left(V^{*}\right)$ whose fiber over $l \in \mathbb{P}_{+}\left(V^{*}\right)$ is equal to the space of $\mathbb{R}$-valued linear functionals on $l$. Let us denote by $\mathcal{H}:=C^{\infty}\left(\mathbb{P}_{+}\left(V^{*}\right), L\right)$ the Fréchet space of $C^{\infty}$-smooth sections of $L$. Clearly $\mathcal{H}$ coincides with the space of smooth functions on $V^{*} \backslash\{0\}$ which are homogeneous of degree one.

3.1.4. Lemma. Consider the maps

$$
\xi: \mathcal{H} \times V \times \mathbb{P}_{+}\left(V^{*}\right) \times[0,1] \rightarrow V
$$

defined by

$$
\xi(h, p, n, t)=p+t \cdot \nabla h(n)
$$

and

$$
\Xi: \mathcal{H} \times C^{\infty}\left(V,\left|\omega_{V}\right|\right) \rightarrow C^{\infty}\left(V \times \mathbb{P}_{+}\left(V^{*}\right) \times[0,1], \Omega^{n} \otimes p^{*} o\right)
$$

defined by

$$
\Xi(h, \eta)=(\xi(h, \cdot))^{*} \eta
$$

where $\xi(h, \cdot): V \times \mathbb{P}_{+}\left(V^{*}\right) \times[0,1] \rightarrow V$ is defined by (26).

Then $\Xi$ is an infinitely smooth map (of infinite dimensional manifolds), and it is linear with respect to the second argument. 
Proof. Is obvious.

For $k \in \mathbb{Z}_{+}$let us denote by $\Xi_{k}, \frac{1}{k !}$ times the $k$-th differential of $\Xi$ at 0 with respect to the first argument. Namely for $h_{1}, \ldots, h_{k} \in \mathcal{H}, \eta \in C^{\infty}(V,|\omega|)$

$$
\Xi_{k}\left(h_{1}, \ldots, h_{k}, \eta\right)=\left.\frac{\partial^{k}}{\partial \lambda_{1} \ldots \partial \lambda_{k}}\right|_{0}\left(\xi\left(\sum_{i=1}^{k} \lambda_{i} h_{i}\right)\right)^{*} \eta .
$$

Thus

$$
\Xi_{k}: \mathcal{H}^{k} \times C^{\infty}\left(V,\left|\omega_{V}\right|\right) \rightarrow C^{\infty}\left(V \times \mathbb{P}_{+}\left(V^{*}\right) \times[0,1], \Omega^{n} \otimes p^{*} o\right)
$$

is a continuous map linear with respect to all $k+1$ arguments.

By the L. Schwartz kernel theorem, $\Xi_{k}$ extends canonically to a continuous linear operator

(27) $\Xi_{k}: C^{\infty}\left(V \times\left(\mathbb{P}_{+}\left(V^{*}\right)\right)^{k},\left|\omega_{V}\right| \otimes L^{\bigotimes k}\right) \rightarrow C^{\infty}\left(V \times \mathbb{P}_{+}\left(V^{*}\right) \times[0,1], \Omega^{n} \otimes p^{*} o\right)$.

(Note that we denote this operator by the same letter $\Xi_{k}$.)

Let us also denote

$$
\Theta: C^{\infty}\left(V \times \mathbb{P}_{+}\left(V^{*}\right) \times[0,1], \Omega^{n} \otimes p^{*} o\right) \rightarrow V^{\infty}(V)
$$

as the canonical map given by integration with respect to the normal cycle times the segment $[0,1]$. Namely $(\Theta(\omega))(P)=\int_{N^{*}(P) \times[0,1]} \omega$ for any $P \in \mathcal{P}(V)$.

3.1.5. Lemma. Let $\psi \in V^{\infty}(V)$. Assume that there exist $k \in \mathbb{N}$, sequences of smooth densities $\left\{\mu_{N}\right\}_{N=1}^{\infty} \subset C^{\infty}\left(V,\left|\omega_{V}\right|\right)$, and $\left\{B_{N}^{i}\right\}_{N=1}^{\infty} \subset \mathcal{K}(V), i=1, \ldots, k$, of strictly convex compact sets with smooth boundaries, containing the origin in the interior, and such that

1) for any compact subset $T \subset V$ and any $L \in \mathbb{N}$

$$
\sum_{N=1}^{\infty}\left\|\mu_{N}\right\|_{C^{L}(T)} \cdot \prod_{i=1}^{k}\left\|h_{B_{N}^{i}}\right\|_{C^{L}\left(S^{n-1}\right)}<\infty ;
$$

2) for any $K \in \mathcal{K}(V) \cap \mathcal{P}(V)$ one has

$$
\psi(K)=\left.\sum_{N=1}^{\infty} \frac{\partial^{k}}{\partial \lambda_{1} \ldots \partial \lambda_{k}}\right|_{0} \mu_{N}\left(K+\sum_{i=1}^{k} \lambda_{i} B_{N}^{i}\right) .
$$

Then one has

$$
\psi=\sum_{N=1}^{\infty}\left(\Theta \circ \Xi_{k}\right)\left(\mu_{N} \otimes h_{B_{N}^{1}} \otimes \cdots \otimes h_{B_{N}^{k}}\right)
$$

where the last series converges in the space $V^{\infty}(V)$.

Proof. The inequality (29) implies that the series $\sum_{N=1}^{\infty} \mu_{N} \otimes h_{B_{N}^{1}} \otimes \cdots \otimes h_{B_{N}^{k}}$ converges in $C^{\infty}\left(V \times\left(\mathbb{P}_{+}\left(V^{*}\right)\right)^{k},\left|\omega_{V}\right| \otimes L^{\bigotimes k}\right)$. Hence the series on the right hand side of $(31)$ converges in $V^{\infty}(V)$ due to the continuity of $\Theta$ and $\Xi_{k}$. Let us denote its limit by $\psi^{\prime}$.

By Lemma 2.4.5 of [5] any smooth valuation is defined uniquely by its values on sets from $\mathcal{K}(V) \cap \mathcal{P}(V)$. Hence it is enough to check that for any $K \in \mathcal{K}(V) \cap \mathcal{P}(V)$ one has

$$
\psi(K)=\psi^{\prime}(K)
$$


By continuity we may assume that

$$
\psi(K)=\left.\frac{\partial^{k}}{\partial \lambda_{1} \ldots \partial \lambda_{k}}\right|_{0} \mu\left(K+\sum_{i=1}^{k} \lambda_{i} B^{i}\right)
$$

for any $K \in \mathcal{K}(V) \cap \mathcal{P}(V)$, and thus $\psi^{\prime}=\left(\Theta \circ \Xi_{k}\right)\left(\mu \otimes h_{B^{1}} \otimes \cdots \otimes h_{B^{k}}\right)$.

Fix $K \in \mathcal{K}(V) \cap \mathcal{P}(V)$. By Lemma 3.1.1 and Proposition 3.1.2 the map $\operatorname{Nor}_{1}(K) \times$ $(0,1] \rightarrow V$ given by

$$
(p, n, t) \mapsto p+t \sum_{i=1}^{k} \lambda_{i} \nabla h_{B^{i}}(n)
$$

is a homeomorphism of $\operatorname{Nor}_{1}(K) \times(0,1]$ onto $\left(K+\sum_{i=1}^{k} \lambda_{i} B^{i}\right) \backslash K$ for $0<\lambda_{1}, \ldots, \lambda_{k}$ $\ll 1$. Hence

$$
\begin{aligned}
\mu\left(K+\sum_{i=1}^{k} \lambda_{i} B^{i}\right) & =\mu(K)+\int_{N^{*}(K) \times(0,1]}\left(\xi\left(\sum_{i=1}^{k} \lambda_{i} \nabla h_{B^{i}}\right)\right)^{*} \mu \\
& =\mu(K)+\int_{N^{*}(K) \times(0,1]} \Xi\left(\sum_{i=1}^{k} \lambda_{i} h_{B^{i}}, \mu\right) .
\end{aligned}
$$

Hence

$$
\begin{aligned}
\psi(K) & =\int_{N^{*}(K) \times[0,1]} \Xi_{k}\left(h_{B^{1}}, \ldots, h_{B^{k}}, \mu\right) \\
& =\left(\left(\Theta \circ \Xi_{k}\right)\left(\mu \otimes h_{B^{1}} \otimes \cdots \otimes h_{B^{k}}\right)\right)(K)=\psi^{\prime}(K) .
\end{aligned}
$$

We will also need the following simple lemma.

3.1.6. Lemma. For any subsets $T \subset V \times V, A, B \subset V$, and any $x_{0} \in V$ one has

$$
(T+(A \times B)) \cap\left(\left\{x_{0}\right\} \times V\right)=\left\{x_{0}\right\} \times\left(p_{2}\left(T \cap p_{1}^{-1}\left(x_{0}-A\right)\right)+B\right) .
$$

Proof. We have

$$
(T+(A \times B)) \cap\left(\left\{x_{0}\right\} \times V\right)=\left((T+(A \times\{0\})) \cap\left(\left\{x_{0}\right\} \times V\right)\right)+\left(\left\{x_{0}\right\} \times B\right) .
$$

Then we have for any $y \in V$

$$
\begin{array}{r}
\left(x_{0}, y\right) \in(T+(A \times\{0\})) \cap\left(\left\{x_{0}\right\} \times V\right) \\
\Leftrightarrow \exists x \in V \exists a \in A \text { s.t. } x+a=x_{0} \text { and }(x, y) \in T \\
\Leftrightarrow \exists x \in V \text { s.t. }(x, y) \in T \cap p_{1}^{-1}\left(x_{0}-A\right) .
\end{array}
$$

The result follows.

In general we will denote by $\|\cdot\|_{0}$ the $C_{0}$-norm of a map.

3.1.7. Lemma. Let $\mathcal{D}, \mathcal{D}^{\prime} \subset V$ be compact domains with smooth boundaries, and let $g: \mathcal{D} \stackrel{\sim}{\rightarrow} \mathcal{D}^{\prime}$ be a diffeomorphism. Let $K \subset \mathcal{D}$ be a compact convex subset, and put $\delta$ for the distance from $K$ to the complement of $\mathcal{D}$. Then

$$
\operatorname{reach} g(K) \geq \min \left\{\frac{\delta}{2}\left\|D\left(g^{-1}\right)\right\|_{0}^{-1},\left\|D\left(g^{-1}\right)\right\|_{0}^{-2}\left\|D^{2} g\right\|_{0}^{-1}\right\} .
$$


Proof. Suppose reach $g(K)<\frac{\delta}{2}\left\|D\left(g^{-1}\right)\right\|_{0}^{-1}$. Let $\varepsilon>0$ be given. Then there exist points $q \in V$ and $x_{0}, x_{1} \in K, x_{0} \neq x_{1}$, such that

$$
\begin{aligned}
& \left|q-g\left(x_{i}\right)\right|=\operatorname{dist}(q, g(K))<\frac{\delta}{2}\left\|D\left(g^{-1}\right)\right\|_{0}^{-1}, \\
& \left|q-g\left(x_{i}\right)\right|<\operatorname{reach} g(K)+\varepsilon, \quad i=0,1 .
\end{aligned}
$$

We may assume for simplicity that $x_{0}=g\left(x_{0}\right)=0$. The mean value theorem implies that the distance from $g(K)$ to the complement of $\mathcal{D}^{\prime}$ is at least $\delta\left\|D\left(g^{-1}\right)\right\|_{0}^{-1}>$ $2|q|$, so by the triangle inequality the line segment joining 0 to $g\left(x_{1}\right)$ lies in $\mathcal{D}^{\prime}$.

Since 0 and $g\left(x_{1}\right)$ both lie on the sphere of radius $|q|$ about $q$, it follows that

$$
\left(q, g\left(x_{1}\right)\right)=\frac{\left|g\left(x_{1}\right)\right|^{2}}{2} \geq \frac{\left|x_{1}\right|^{2}}{2}\left\|D\left(g^{-1}\right)\right\|_{0}^{-2}
$$

by the mean value theorem. Abbreviating $L:=D g(0)$ and letting $\hat{L}$ be its adjoint, it is clear that $\hat{L}(q) \in \operatorname{Nor}(K, 0)$. Thus by $(33)$,

$$
\begin{aligned}
\frac{\left|x_{1}\right|^{2}}{2}\left\|D\left(g^{-1}\right)\right\|_{0}^{-2} & \leq\left(L\left(x_{1}\right), q\right)+\left(g\left(x_{1}\right)-L\left(x_{1}\right), q\right) \\
& =\left(x_{1}, \hat{L}(q)\right)+\left(g\left(x_{1}\right)-L\left(x_{1}\right), q\right) \\
& \leq\left(g\left(x_{1}\right)-L\left(x_{1}\right), q\right) \\
& \leq \frac{\left|x_{1}\right|^{2}}{2}\left\|D^{2} g\right\|_{0}|q|
\end{aligned}
$$

by Taylor's theorem and the Cauchy-Schwartz inequality. Thus

$$
\operatorname{reach} g(K)+\varepsilon>|q| \geq\left\|D\left(g^{-1}\right)\right\|_{0}^{-2}\left\|D^{2} g\right\|_{0}^{-1} .
$$

3.1.8. Lemma. Let $X \subset X^{\prime} \subset V \times V$ be submanifolds as in the beginning of this section. Assume moreover that $p_{1}(X)$ is convex.

Then there exists $\delta>0$ (depending on the $C^{2}$-norm of the map $\left.\left(p_{2} \circ p_{1}^{-1}\right)\right|_{p_{1}\left(X^{\prime}\right)}$ and its inverse, and the distance from $X$ to $\partial X^{\prime}$ only) such that for any $A \in \mathcal{K}(V)$ and any $x \in V$ the set $(X+(A \times\{0\})) \cap(\{x\} \times V)$ is either empty or has reach at least $\delta$ as a subset of $\{x\} \times V$.

Proof. Lemma 3.1.6 and the assumptions imply that

$$
(X+(A \times\{0\})) \cap\{x\} \times V=\{x\} \times\left(\tilde{p}_{2} \circ \tilde{p}_{1}^{-1}\right)\left(p_{1}(X) \cap(x-A)\right) .
$$

Now the proof follows immediately from Lemma 3.1.7.

From Lemmas 3.1.6 and 3.1.8 we immediately deduce the following corollary.

3.1.9. Corollary. Let $X \subset X^{\prime} \subset V \times V$ be as at the beginning of this section. Assume that $p_{1}(X)$ is convex.

Then for any $x \in V$ and any $\psi \in V^{\infty}(V)$ one has

$$
\psi((X+(A \times\{0\})) \cap(\{x\} \times V))=\left(\left(\tilde{p}_{1} \circ \tilde{p}_{2}^{-1}\right)_{*} \psi\right)\left(p_{1}(X) \cap(x-A)\right)
$$

where $\tilde{p}_{1}, \tilde{p}_{2}: X^{\prime} \rightarrow V$ are the restrictions of the projections $p_{1}, p_{2}$ to $X^{\prime}$.

3.1.10. Lemma. (1) The function

$$
\gamma: V^{\infty}(V) \times C^{\infty}\left(V,\left|\omega_{V}\right|\right) \times \mathcal{K}(V)^{k+1} \times \mathbb{R}_{\geq 0}^{k} \rightarrow \mathbb{C}
$$


defined by

$$
\left(\phi ; \mu ; K, A^{1}, \ldots, A^{k} ; \lambda_{1}, \ldots, \lambda_{k}\right) \stackrel{\gamma}{\mapsto} \int_{x \in V} \phi\left(K \cap\left(x-\sum_{i=1}^{k} \lambda_{i} A^{i}\right)\right) d \mu(x)
$$

with $\phi \in V^{\infty}(V), \mu \in C^{\infty}(V,|\omega|),\left(K, A^{1}, \ldots, A^{k}\right) \in \mathcal{K}(V)^{k+1}, \lambda_{i} \geq 0$, is a continuous function which is $C^{\infty}$-smooth on $V^{\infty}(V) \times C^{\infty}(V,|\omega|) \times \mathbb{R}_{\geq 0}^{k}$ for fixed $\left(K, A^{1}, \ldots, A^{k}\right) \in \mathcal{K}(V)^{k+1}$

(2) Fix $R>0, k \in \mathbb{N}$. Then there exist a constant $C$, a positive integer $L \in \mathbb{N}$, and continuous seminorms $\|\cdot\|$ and $\|\cdot\|^{\prime}$ on $V^{\infty}(V)$ and $C^{\infty}\left(V,\left|\omega_{V}\right|\right)$ respectively depending on $n, k$, and $R$ only, such that for any strictly convex compact sets $A^{1}, \ldots, A^{k}$ with smooth boundaries, and any $K \in \mathcal{K}(V)$ such that $K$ is contained in the centered Euclidean ball of radius $R$, one has an estimate

$$
\left|\frac{\partial^{k}}{\partial \lambda_{1} \ldots \partial \lambda_{k}}\right|_{0} \int_{x \in V} \phi\left(K \cap\left(x-\sum_{i=1}^{k} \lambda_{i} A^{i}\right)\right) d \mu(x) \mid \leq\|\phi\| \cdot\|\mu\|^{\prime} \cdot \prod_{i=1}^{k}\left\|h_{A^{i}}\right\|_{C^{L}\left(S^{n-1}\right)} .
$$

Proof. By Theorem 1.3.2 there exists a continuous epimorphism of Fréchet spaces

$$
\mathcal{T}: \bigoplus_{k=0}^{n} C^{\infty}\left(V \times \mathbb{P}_{+}\left(V^{*}\right)^{k},\left|\omega_{V}\right| \otimes L^{\bigotimes k}\right) \rightarrow S V(V)\left(\tilde{\leftarrow} V^{\infty}(V)\right)
$$

which is uniquely characterized by the property that for any $K \in \mathcal{K}(V)$, any strictly convex compact sets with smooth boundary $A_{1}, \ldots, A_{k}$, and any $\nu \in C^{\infty}\left(V,\left|\omega_{V}\right|\right)$ one has

$$
\mathcal{T}\left(\nu \otimes h_{A_{1}} \otimes \cdots \otimes h_{A_{k}}\right)(K)=\left.\frac{\partial^{k}}{\partial \lambda_{1} \ldots \partial \lambda_{k}}\right|_{0} \nu\left(K+\sum_{i=1}^{k} \lambda_{i} A_{i}\right) .
$$

By the Banach inverse mapping theorem the map $\mathcal{T}$ induces an isomorphism of Fréchet spaces

$$
\left(\bigoplus_{k=0}^{n} C^{\infty}\left(V \times \mathbb{P}_{+}\left(V^{*}\right)^{k},\left|\omega_{V}\right| \otimes L^{\otimes k}\right)\right) / \operatorname{Ker} \mathcal{T} \stackrel{\sim}{\rightarrow} S V(V)\left(\tilde{\leftarrow} V^{\infty}(V) .\right.
$$

Let us consider the composition of $\gamma$ with $\mathcal{T}$. Thus for any $0 \leq l \leq n$ we get a map

$$
\mathcal{T}_{l}^{\prime}: C^{\infty}\left(V \times \mathbb{P}_{+}\left(V^{*}\right)^{l},\left|\omega_{V}\right| \otimes L^{\otimes l}\right) \times C^{\infty}\left(V,\left|\omega_{V}\right|\right) \times \mathcal{K}(V)^{k+1} \times \mathbb{R}_{\geq 0}^{k} \rightarrow \mathbb{C} .
$$

Also consider the canonical $(l+1)$-linear map

$$
C^{\infty}(V,|\omega|) \times\left(C^{\infty}\left(\mathbb{P}_{+}(V), L\right)\right)^{l} \rightarrow C^{\infty}\left(V \times \mathbb{P}_{+}\left(V^{*}\right)^{l},|\omega| \otimes L^{\otimes l}\right) .
$$

Composing $\mathcal{T}_{l}^{\prime}$ with the map (36) we get the map

(37) $\mathcal{T}_{l}^{\prime \prime}: C^{\infty}(V,|\omega|) \times\left(C^{\infty}\left(\mathbb{P}_{+}\left(V^{*}\right), L\right)\right)^{l} \times C^{\infty}(V,|\omega|) \times \mathcal{K}(V)^{k+1} \times \mathbb{R}_{\geq 0}^{k} \rightarrow \mathbb{C}$

which is uniquely characterized by the following property: for any $\mu, \nu \in$ $C^{\infty}\left(V,\left|\omega_{V}\right|\right)$, any $B^{1}, \ldots, B^{l} \in \mathcal{K}(V)$ being strictly convex compact sets with smooth boundaries, and any $K, A^{1}, \ldots, A^{k} \in \mathcal{K}(V)$ one has

$$
\begin{array}{r}
\mathcal{T}_{l}^{\prime \prime}\left(\nu ; h_{B^{1}}, \ldots, h_{B^{l}} ; \mu ; K ; A^{1}, \ldots, A^{k} ; \lambda_{1}, \ldots, \lambda_{k}\right) \\
=\left.\int_{x \in V} \frac{\partial^{l}}{\partial \mu_{1} \ldots \partial \mu_{l}}\right|_{0} \nu\left(\left(K \cap\left(x-\sum_{i=1}^{k} \lambda_{i} A^{i}\right)\right)+\sum_{j=1}^{l} \mu_{j} B^{j}\right) d \mu(x) .
\end{array}
$$


Using the L. Schwartz kernel theorem it is easy to see that in order to prove Lemma 3.1.10 it is enough to show that for any $0 \leq l \leq n$ the map $\mathcal{T}_{l}^{\prime \prime}$ has the following properties:

(1) $\mathcal{T}_{l}^{\prime \prime}$ is $C^{\infty}$-smooth for fixed $K, A^{1}, \ldots, A^{k}$;

(2) for any $R>0$ there exists a continous semi-norm $\|\cdot\|$ on $C^{\infty}\left(V,\left|\omega_{V}\right|\right)$ such that for any $K \in \mathcal{K}(V)$ contained in the origin symmetric Euclidean ball of radius $R$, and any strictly convex compact sets with smooth boundaries $B_{1}, \ldots, B_{l} \in \mathcal{K}(V)$ one has an estimate

$$
\begin{array}{r}
\left|\frac{\partial^{k}}{\partial \lambda_{1} \ldots \partial \lambda_{k}}\right|{ }_{0} \mathcal{T}_{l}^{\prime \prime}\left(\nu ; B_{1}, \ldots, B_{l} ; \mu ; K, A_{1}, \ldots, A_{k} ; \lambda_{1}, \ldots, \lambda_{k}\right) \mid \\
\leq\|\nu\| \cdot\|\mu\| \cdot \prod_{i=1}^{k}\left\|h_{A_{i}}\right\|_{C^{2}\left(\mathbb{P}_{+}\left(V^{*}\right)\right)} \cdot \prod_{j=1}^{l}\left\|h_{B_{j}}\right\|_{C^{2}\left(\mathbb{P}_{+}\left(V^{*}\right)\right)}
\end{array}
$$

In order to prove the last inequality let us observe that for fixed $\lambda_{1}, \ldots, \lambda_{k} \geq 0$

$$
\begin{gathered}
\int_{x \in V} \nu\left(K \cap\left(x-\sum_{i=1}^{k} \lambda_{i} A_{i}\right)+\sum_{j=1}^{l} \mu_{j} B_{j}\right) d \mu(x) \\
=(\mu \otimes \nu)\left(\Delta(K)+\left(\sum_{i=1}^{k} \lambda_{i} A_{i}, \sum_{j=1}^{l} \mu_{j} B_{j}\right)\right)
\end{gathered}
$$

where $\Delta: V \hookrightarrow V \times V$ is the diagonal imbedding. Now the inequality follows from Corollary 1.2.2. Corollary 1.2 .2 also implies the smoothness of $\mathcal{T}_{l}^{\prime \prime}$.

3.1.11. Lemma. Let $X \subset X^{\prime} \subset V \times V$ be as at the beginning of this section. Assume that $p_{1}(X)$ is convex. Let $A \in \mathcal{K}(V)$. Let

$$
\psi=\left(\Theta \circ \Xi_{l}\right)\left(\nu \otimes h_{B^{1}} \otimes \cdots \otimes h_{B^{l}}\right),
$$

where $B^{1}, \ldots, B^{l}$ are strictly convex compact sets with smooth boundaries and containing the origin in their interiors. Let $\mu, \nu$ be smooth densities on $V$. Then there exists $\varepsilon>0$ depending on $X$ and $X^{\prime}$ only such that the function $f:[0, \varepsilon]^{l} \rightarrow \mathbb{C}$ defined by

$$
f\left(\mu_{1}, \ldots, \mu_{l}\right):=(\mu \otimes \nu)\left(X+\left(A \times\left(\sum_{i=1}^{l} \mu_{i} B^{i}\right)\right)\right)
$$

is $C^{\infty}$-smooth. Moreover

$$
\left.\frac{\partial^{l}}{\partial \mu_{1} \ldots \partial \mu_{l}}\right|_{0} f\left(\mu_{1}, \ldots, \mu_{l}\right)=\int_{x \in V}\left(\left(\tilde{p}_{1} \circ \tilde{p}_{2}^{-1}\right)_{*} \psi\right)\left(p_{1}(X) \cap(x-A)\right) d \mu(x) .
$$

Proof. Let us choose $\varepsilon>0$ such that for any $x \in V$ the map

$$
N((X+A \times\{0\}) \cap(\{x\} \times V)) \times(0,1] \rightarrow V
$$

given by $(p, n, t) \mapsto p+t \sum_{i=1}^{l} \mu_{i} \nabla h_{B^{i}}(n)$ is a homeomorphism of

$$
N((X+A \times\{0\}) \cap(\{x\} \times V)) \times(0,1]
$$

onto its image

$$
\left(p_{2}((X+A \times\{0\}) \cap(\{x\} \times V))+\sum_{i=1}^{l} \mu_{i} B^{i}\right) \backslash\left(p_{2}((X+A \times\{0\}) \cap(\{x\} \times V))\right)
$$


for $0<\mu_{1}, \ldots, \mu_{l} \leq \varepsilon$. Such an $\varepsilon$ exists due to Lemmas 3.1.8, 3.1.1, and Proposition 3.1.2.

Let us denote

$$
f\left(\mu_{1}, \ldots, \mu_{l}\right):=(\mu \otimes \nu)\left(X+\left(A \times\left(\sum_{i=1}^{l} \mu_{i} B^{i}\right)\right)\right) .
$$

We have

$$
\begin{array}{r}
f\left(\mu_{1}, \ldots, \mu_{l}\right) \\
=\int_{x \in V} d \mu(x) \nu\left(((X+A \times\{0\}) \cap(\{x\} \times V))+\{x\} \times\left(\sum_{i=1}^{l} \mu_{i} B^{i}\right)\right) \\
=\int_{x \in V} d \mu(x) \int_{N((X+A \times\{0\}) \cap(\{x\} \times V)) \times[0,1]}\left(\xi\left(\sum_{i=1}^{l} \mu_{i} h_{B^{i}}, \cdot\right)\right)^{*} \nu \\
=\int_{x \in V} d \mu(x) \int_{N((X+A \times\{0\}) \cap(\{x\} \times V)) \times[0,1]} \eta_{\mu_{1}, \ldots, \mu_{l}}
\end{array}
$$

where $\eta_{\mu_{1}, \ldots, \mu_{l}}:=\left(\xi\left(\sum_{i=1}^{l} \mu_{i} h_{B^{i}}, \cdot\right)\right)^{*} \nu$. Consider the natural projection

$$
q: V \times \mathbb{P}_{+}\left(V^{*}\right) \times[0,1] \rightarrow V \times \mathbb{P}_{+}\left(V^{*}\right) .
$$

Set

$$
\tilde{\eta}_{\mu_{1}, \ldots, \mu_{l}}:=q_{*} \eta_{\mu_{1}, \ldots, \mu_{l}} \in C^{\infty}\left(V \times \mathbb{P}_{+}\left(V^{*}\right), \Omega^{n-1} \otimes p^{*} o\right)
$$

(here $q_{*}$ denotes integration along the fibers). Using Lemma 2.2.1 and the relation (34), we compute

$$
\begin{aligned}
& f\left(\mu_{1}, \ldots, \mu_{l}\right) \\
& =\int_{x \in V} d \mu(x) \int_{N^{*}((X+(A \times\{0\})) \cap(\{x\} \times V))} \tilde{\eta}_{\mu_{1}, \ldots, \mu_{l}} \\
& =\int_{x \in V} d \mu(x) \int \frac{}{\left(\tilde{p}_{1} \circ \tilde{p}_{2}^{-1}\right) * N^{*}\left(p_{1}(X) \cap(x-A)\right)} \tilde{\eta}_{\mu_{1}, \ldots, \mu_{l}} \\
& =\int_{x \in V} d \mu(x) \int_{N^{*}\left(p_{1}(X) \cap(x-A)\right)}{\overline{\left(\tilde{p}_{1} \circ \tilde{p}_{2}^{-1}\right)}}^{*} \tilde{\eta}_{\mu_{1}, \ldots, \mu_{l}} .
\end{aligned}
$$

Here $\overline{\left(\tilde{p}_{1} \circ \tilde{p}_{2}^{-1}\right)}$ denotes the natural lift of the diffeomorphism $\tilde{p}_{1} \circ \tilde{p}_{2}^{-1}$ to $V \times$ $\mathbb{P}_{+}\left(V^{*}\right)$. It is easy to see that the map $\mathbb{R}^{l} \rightarrow C^{\infty}\left(V \times \mathbb{P}_{+}\left(V^{*}\right), \Omega^{n-1} \otimes p^{*} o\right)$ given by $\left(\mu_{1}, \ldots, \mu_{l}\right) \mapsto \tilde{\eta}_{\mu_{1}, \ldots, \mu_{l}}$ is $C^{\infty}$-smooth. This and Lemma 3.1.10(1) imply the first statement of the lemma.

Let us prove the second statement. Observe that for any compact semi-convex (= positive reach) subset $Y \subset V$

$$
\psi(Y)=\left.\frac{\partial^{l}}{\partial \mu_{1} \ldots \partial \mu_{l}}\right|_{0} \int_{N(Y)} \tilde{\eta}_{\mu_{1}, \ldots, \mu_{l}} .
$$

Hence

$$
\left.\frac{\partial^{l}}{\partial \mu_{1} \ldots \partial \mu_{l}}\right|_{0} f\left(\mu_{1}, \ldots, \mu_{l}\right)=\int_{x \in V} d \mu(x)\left(\left(\tilde{p}_{1} \circ \tilde{p}_{2}^{-1}\right)_{*} \psi\right)\left(p_{1}(X) \cap(x-A)\right) .
$$


3.1.12. Lemma. Let $\psi \in V^{\infty}(V)$ be a smooth valuation of the form

$$
\psi=\left(\Theta \circ \Xi_{l}\right)\left(\sum_{N=1}^{\infty} \nu_{N} \otimes h_{B_{N}^{1}} \otimes \cdots \otimes h_{B_{N}^{l}}\right)
$$

with $\left\{\nu_{N}\right\} \subset C^{\infty}\left(V,\left|\omega_{V}\right|\right)$ being smooth densities, and $B_{N}^{i} \in \mathcal{K}(V)$ being strictly convex compact sets with smooth boundaries, containing the origin in the interior, and such that for any compact subset $T \subset V$ and any $L \in \mathbb{N}$

$$
\sum_{N=1}^{\infty}\left\|\nu_{N}\right\|_{C^{L}(T)} \prod_{i=1}^{l}\left\|h_{B_{N}^{i}}\right\|_{C^{L}\left(S^{n-1}\right)}<\infty .
$$

Let $A \in \mathcal{K}(V)$. Let $\mu \in C^{\infty}\left(V,\left|\omega_{V}\right|\right)$. Let $X \subset X^{\prime} \subset V \times V$ be as in the beginning of this section. Assume that $p_{1}(X)$ is convex.

Then the series

$$
\left.\sum_{N=1}^{\infty} \frac{\partial^{l}}{\partial \mu_{1} \ldots \partial \mu_{l}}\right|_{0}\left(\mu \otimes \nu_{N}\right)\left(X+A \times\left(\sum_{i=1}^{l} \mu_{i} B_{N}^{i}\right)\right)
$$

converges absolutely and its sum is equal to

$$
\int_{x \in V}\left(\left(\tilde{p}_{1} \circ \tilde{p}_{2}^{-1}\right)_{*} \psi\right)\left(p_{1}(X) \cap(x-A)\right) d \mu(x)
$$

where as previously $\tilde{p}_{1}, \tilde{p}_{2}: X^{\prime} \rightarrow V$ are the restrictions of the projections $p_{1}, p_{2}$ to $X^{\prime}$.

Proof. If the sum in (46) is finite, then the statement follows immediately from Lemma 3.1.11. Next let us observe that the expression (49) is continuous with respect to $\psi \in V^{\infty}(V)$. Hence it is enough to check that the series (48) converges absolutely.

Let us denote

$$
\psi_{N}:=\left(\Theta \circ \Xi_{l}\right)\left(\nu_{N} \otimes h_{B_{N}^{1}} \otimes \cdots \otimes h_{B_{N}^{l}}\right) .
$$

By Lemma 3.1.11 we have

$$
\begin{aligned}
& \left.\sum_{N=1}^{\infty} \frac{\partial^{l}}{\partial \mu_{1} \ldots \partial \mu_{l}}\right|_{0}\left(\mu \otimes \nu_{N}\right)\left(X+A \times\left(\sum_{i=1}^{l} \mu_{i} B_{N}^{i}\right)\right) \\
= & \sum_{N=1}^{\infty} \int_{x \in V}\left(\left(\tilde{p}_{1} \circ \tilde{p}_{2}^{-1}\right)_{*} \psi_{N}\right)\left(p_{1}(X) \cap(x-A)\right) d \mu(x) .
\end{aligned}
$$

It follows from the assumption (47) that the series $\sum_{N=1}^{\infty} \psi_{N}$ converges absolutely in $V^{\infty}(V)$. Hence the series $\sum_{N=1}^{\infty}\left(\tilde{p}_{1} \circ \tilde{p}_{2}^{-1}\right)_{*} \psi_{N}$ converges absolutely in $V^{\infty}\left(p_{1}\left(X^{\prime}\right)\right)$.

3.1.13. Proposition. Let $X \subset X^{\prime} \subset V \times V$ be as at the beginning of this section. Assume moreover that $p_{1}(X)$ and $p_{2}(X)$ are convex. Fix $\delta>0$. Then there exists $\varepsilon_{0}>0$ depending on $\delta$, the $C^{2}$-norm of the map $\left.\left(p_{2} \circ p_{1}^{-1}\right)\right|_{p_{1}\left(X^{\prime}\right)}$ and its inverse, and the distance from $X$ to $\partial X^{\prime}$ only such that the following properties are satisfied.

Let $\mathcal{A}=\left(A^{1}, \ldots, A^{k}\right)$ and $\mathcal{B}=\left(B^{1}, \ldots, B^{l}\right)$ be any $k$ - and l-tuples respectively of strictly convex compact subsets in $V$ with smooth boundaries, principal curvatures between $\delta$ and $1 / \delta$, and containing the origin in their interiors. Let $\mu, \nu$ be any smooth densities on $V$. 
(1) Then the function

$$
f_{\mathcal{A}, \mathcal{B}}\left(\lambda_{1}, \ldots, \lambda_{k} ; \mu_{1}, \ldots, \mu_{l}\right):=(\mu \nabla \nu)\left(X+\left(\sum_{i=1}^{k} \lambda_{i} A^{i}, \sum_{j=1}^{l} \mu_{j} B^{j}\right)\right)
$$

is $C^{\infty}$-smooth for $\left(\lambda_{1}, \ldots, \lambda_{k} ; \mu_{1}, \ldots, \mu_{l}\right) \in[0, \varepsilon]^{k+l}$ for any $0<\varepsilon<\varepsilon_{0}$ and such that $p_{1}(X)+\varepsilon \sum_{i} A^{i} \subset p_{1}\left(X^{\prime}\right)$ and $p_{2}(X)+\varepsilon \sum_{j} B^{j} \subset p_{2}\left(X^{\prime}\right)$.

(2) There exist a continuous semi-norm $\|\cdot\|$ on $C^{\infty}\left(V,\left|\omega_{V}\right|\right)$ and a positive integer $L \in \mathbb{N}$ depending on $X, X^{\prime}, k, l$ only (and independent of $\mathcal{A}, \mathcal{B}, \mu, \nu$ ) such that

$$
\begin{aligned}
& \left|\frac{\partial^{k+l}}{\partial \lambda_{1} \ldots \partial \lambda_{k} \partial \mu_{1} \ldots \partial \mu_{l}}\right|_{0} f_{\mathcal{A}, \mathcal{B}}\left(\lambda_{1}, \ldots, \lambda_{k} ; \mu_{1}, \ldots, \mu_{l}\right) \mid \\
\leq & C\|\mu\| \cdot\|\nu\| \cdot \prod_{i=1}^{k}\left\|h_{A^{i}}\right\|_{C^{L}\left(S^{n-1}\right)} \cdot \prod_{j=1}^{l}\left\|h_{B^{j}}\right\|_{C^{L}\left(S^{n-1}\right)} .
\end{aligned}
$$

Proof. Similar to the proof of Lemma 3.1.11, let us denote

$$
\begin{gathered}
\eta_{\mathcal{B}, \mu_{1}, \ldots, \mu_{l}}:=\left(\xi\left(\sum_{i=1}^{l} \mu_{i} h_{B^{i}}, \cdot\right)\right)^{*} \nu \in C^{\infty}\left(V \times \mathbb{P}_{+}\left(V^{*}\right) \times[0,1], \Omega^{n} \otimes p^{*} o\right) \\
\tilde{\eta}_{\mathcal{B}, \mu_{1}, \ldots, \mu_{l}}:=q_{*} \eta_{\mathcal{B}, \mu_{1}, \ldots, \mu_{l}} \in C^{\infty}\left(V \times \mathbb{P}_{+}\left(V^{*}\right), \Omega^{n-1} \otimes p^{*} o\right),
\end{gathered}
$$

where as previously $q: V \times \mathbb{P}_{+}\left(V^{*}\right) \times[0,1] \rightarrow V \times \mathbb{P}_{+}\left(V^{*}\right)$ is the projection, and $q_{*}$ denotes the integration along the fibers. First let us prove part (1) of the proposition. Exactly as in (45) we have

$$
\begin{aligned}
& f_{\mathcal{A}, \mathcal{B}}\left(\lambda_{1}, \ldots, \lambda_{k} ; \mu_{1}, \ldots, \mu_{l}\right) \\
& =\int_{x \in V} d \mu(x) \int_{N\left(p_{1}(X) \cap\left(x-\sum_{i} \lambda_{i} A^{i}\right)\right)}{\overline{\left(\tilde{p}_{2} \circ \tilde{p}_{1}^{-1}\right)}}^{*} \tilde{\eta}_{\mathcal{B}, \mu_{1}, \ldots, \mu_{l}} .
\end{aligned}
$$

Lemma 3.1.4 implies that $\tilde{\eta}_{\mathcal{B}, \mu_{1}, \ldots, \mu_{l}}$ depends smoothly on $\left(\mu_{1}, \ldots, \mu_{l}\right) \in[0, \varepsilon]^{l}$ and on $h_{B^{1}}, \ldots, h_{B^{l}} \in C^{\infty}\left(S^{n-1}\right)$. Hence

$$
\zeta_{\mathcal{B}, \mu_{1}, \ldots, \mu_{l}}:={\overline{\left(\tilde{p}_{2} \circ \tilde{p}_{1}^{-1}\right)}}^{*} \tilde{\eta}_{\mathcal{B}, \mu_{1}, \ldots, \mu_{l}}
$$

also depends smoothly on $\left(\mu_{1}, \ldots, \mu_{l}\right) \in[0, \varepsilon]^{l}$ and on $h_{B^{1}}, \ldots, h_{B^{l}} \in C^{\infty}\left(S^{n-1}\right)$. Thus

$$
f_{\mathcal{A}, \mathcal{B}}\left(\lambda_{1}, \ldots, \lambda_{k} ; \mu_{1}, \ldots, \mu_{l}\right)=\int_{x \in V} d \mu(x) \int_{N\left(p_{1}(X) \cap\left(x-\sum_{i} \lambda_{i} A^{i}\right)\right)} \zeta_{\mathcal{B}, \mu_{1}, \ldots, \mu_{l}} .
$$

Then by Lemma 3.1.10(1) the function $f_{\mathcal{A}, \mathcal{B}}$ is $C^{\infty}$-smooth in $\left(\lambda_{1}, \ldots, \lambda_{k} ; \mu_{1}, \ldots, \mu_{l}\right)$ $\in[0, \varepsilon]^{k+l}$. This proves part (1) of the proposition.

Let us prove part (2). Let us denote that

$$
\sigma_{\mathcal{B}}:=\left.\frac{\partial^{l}}{\partial \mu_{1} \ldots \partial \mu_{l}}\right|_{0} \zeta_{\mathcal{B}, \mu_{1}, \ldots, \mu_{l}}
$$

Then $\sigma_{\mathcal{B}} \in C^{\infty}\left(V \times \mathbb{P}_{+}\left(V^{*}\right), \Omega^{n-1} \otimes p^{*} o\right)$. Moreover $\sigma_{\mathcal{B}}$ depends continuously and linearly on each $h_{B^{i}} \in C^{\infty}\left(S^{n-1}\right)$. Then for any $M \in \mathbb{N}$ there exist a compact subset $T^{\prime} \subset V, L \in \mathbb{N}$, and a constant $C$ such that

$$
\left\|\sigma_{\mathcal{B}}\right\|_{C^{M}\left(p_{1}\left(X^{\prime}\right)\right)} \leq C\|\nu\|_{C^{L}\left(T^{\prime}\right)} \cdot \prod_{j=1}^{l}\left\|h_{B^{j}}\right\|_{C^{L}\left(S^{n-1}\right)} .
$$


We have

$$
\left.\frac{\partial^{l}}{\partial \mu_{1} \ldots \partial \mu_{l}}\right|_{0} f_{\mathcal{A}, \mathcal{B}}\left(\lambda_{1}, \ldots, \lambda_{k} ; \mu_{1}, \ldots, \mu_{l}\right)=\int_{x \in V} d \mu(x) \int_{N\left(p_{1}(X) \cap\left(x-\sum_{i} \lambda_{i} A^{i}\right)\right)} \sigma_{\mathcal{B}}
$$

(note that the differentiation under the the integral is possible due to Lemma 3.1.10(1)).

Hence by Lemma 3.1.10(2) there exist continuous semi-norms $\|\cdot\|,\|\cdot\|^{\prime}$ on $C^{\infty}\left(p_{1}\left(X^{\prime}\right) \times \mathbb{P}_{+}\left(V^{*}\right), \Omega^{n-1} \otimes p^{*} o\right)$ and $C^{\infty}\left(V,\left|\omega_{V}\right|\right)$ respectively and $L^{\prime} \in \mathbb{N}$ such that

$$
\begin{aligned}
& \left|\frac{\partial^{k+l}}{\partial \lambda_{1} \ldots \lambda_{k} \partial \mu_{1} \ldots \partial \mu_{l}}\right|_{0} f_{\mathcal{A}, \mathcal{B}}\left(\lambda_{1}, \ldots, \lambda_{k} ; \mu_{1}, \ldots, \mu_{l}\right) \mid \\
& \leq\left\|\sigma_{\mathcal{B}}\right\| \cdot\|\mu\|^{\prime} \cdot \prod_{i=1}^{k}\left\|h_{A^{i}}\right\|_{C^{L^{\prime}\left(S^{n-1}\right)}} \\
& \stackrel{\text { by }}{\leq} \mathrm{(54)} C\|\nu\|_{C^{L}\left(T^{\prime}\right)} \cdot\|\mu\|^{\prime} \cdot \prod_{i=1}^{k}\left\|h_{A^{i}}\right\|_{C^{L^{\prime}\left(S^{n-1}\right)}} \cdot \prod_{j=1}^{l}\left\|h_{B^{j}}\right\|_{C^{L}\left(S^{n-1}\right)} .
\end{aligned}
$$

Note that the semi-norms $\|\cdot\|,\|\cdot\|^{\prime}$, and the constant $C$ in (57) are independent of $\mathcal{A}, \mathcal{B}$. This proves part (2) of the proposition.

3.1.14. Lemma. Let $Y$ be a smooth n-dimensional manifold. Let $f_{1}, f_{2}: Y \rightarrow \mathbb{R}^{n}$ be two smooth maps which map $Y$ diffeomorphically onto open subsets $f_{1}(Y), f_{2}(Y) \subset$ $\mathbb{R}^{n}$. Let $\phi \in V^{\infty}(Y)$. Assume that $\phi(K)=0$ for any compact domain $K \subset Y$ with smooth boundary such that both $f_{1}(K)$ and $f_{2}(K)$ are convex.

Then $\phi \equiv 0$.

Proof. Suppose that $\phi \not \equiv 0$. Let $i$ be the integer such that $\phi \in W_{i} \backslash W_{i+1}$.

Let $K \subset Y$ be a compact domain with smooth boundary such that $f_{1}(K) \subset \mathbb{R}^{n}$ is strictly convex. Let us show that for any $y_{0} \in Y$ the set $f_{2}\left(f_{1}^{-1}\left(f_{1}\left(y_{0}\right)+\varepsilon f_{1}(K)\right)\right)$ is convex for $0<\varepsilon \ll 1$. For simplicity of the notation and without loss of generality we may assume that $Y=f_{1}(Y)$ and $f_{1}$ is the identity imbedding. Also we may and will assume that $f_{2}(y)=y+O\left(\left|y-y_{0}\right|^{2}\right)$ as $y \rightarrow y_{0}$, and $y_{0}=0$. Then

$$
\frac{f_{2}(\varepsilon y)}{\varepsilon}=y+O(\varepsilon) \text { as } \varepsilon \rightarrow 0 .
$$

But since $K$ is strictly convex it is clear from the last formula that $\frac{f_{2}(\varepsilon K)}{\varepsilon}$ is also convex for $0<\varepsilon \ll 1$.

Now let us deduce the lemma. Fix $K \subset Y$ a compact domain with smooth boundary such that $f_{1}(K)$ is strictly convex. For

$$
0<\varepsilon \ll 1
$$

the set $f_{2}\left(f_{1}^{-1}\left(f_{1}\left(y_{0}\right)+\varepsilon f_{1}(K)\right)\right)$ is convex. Hence the assumption of the lemma implies that

$$
\lim _{\varepsilon \rightarrow+0} \frac{1}{\varepsilon^{i}} \phi\left(f_{1}^{-1}\left(f_{1}\left(y_{0}\right)+\varepsilon f_{1}(K)\right)\right)=0
$$

Hence $\phi \in W_{i+1}$. This is a contradiction. 


\section{Construction of the product}

Let $X$ be a smooth $n$-dimensional manifold. Let $\phi, \psi \in V^{\infty}(X)$. We are going to present a construction of a product $\phi \cdot \psi$ which a priori will depend on some choices, and then we will show that the product is in fact independent of these choices.

Let $U \subset X$ be an open subset. Let $f: U \stackrel{\sim}{\rightarrow} \mathbb{R}^{n}$ be a diffeomorphism. It was shown in [4], Corollary 3.1.7, that $f_{*} \phi$ can be written (non-uniquely) as

$$
f_{*} \phi=\phi_{0}+\cdots+\phi_{n}
$$

with $\phi_{j} \in W_{n-j}\left(\mathbb{R}^{n}\right)$, and there exist sequences

$$
\begin{array}{r}
\left\{\mu_{N}^{j}\right\}_{N=1}^{\infty} \subset C^{\infty}\left(\mathbb{R}^{n},\left|\omega_{\mathbb{R}^{n}}\right|\right), 0 \leq j \leq n, \\
\left\{A_{N}^{i j}\right\}_{N=1}^{\infty} \subset \mathcal{K}\left(\mathbb{R}^{n}\right), 1 \leq i \leq j \leq n,
\end{array}
$$

being strictly convex compact domains with smooth boundaries and containing 0 in their interiors such that

$\left(1_{f_{*} \phi}\right)$ for any compact subset $T \subset \mathbb{R}^{n}$, any $L \in \mathbb{N}$, and any $0 \leq j \leq n$ one has

$$
\sum_{N=1}^{\infty}\left\|\mu_{N}^{j}\right\|_{C^{L}(T)} \prod_{i=1}^{j}\left\|h_{A_{N}^{i j}}\right\|_{C^{L}\left(S^{n-1}\right)}<\infty ;
$$

$\left(2_{f_{*} \phi}\right)$ for any set $S \in \mathcal{K}\left(\mathbb{R}^{n}\right)$ and any $0 \leq j \leq n$ one has

$$
\phi_{j}(S)=\left.\sum_{N=1}^{\infty} \frac{\partial^{j}}{\partial \lambda_{1} \ldots \partial \lambda_{j}}\right|_{0} \mu_{N}^{j}\left(S+\sum_{i=1}^{j} \lambda_{i} A_{N}^{i j}\right) .
$$

The last expression is well defined since by Corollary 1.2.2 the function $\mu_{N}^{j}\left(S+\sum_{i=1}^{j} \lambda_{i} A_{N}^{i j}\right)$ is $C^{\infty}$-smooth in $\lambda_{1}, \ldots, \lambda_{j} \geq 0$ and the series (62) converges absolutely.

Similarly one can write

$$
f_{*} \psi=\psi_{0}+\cdots+\psi_{n}
$$

with $\psi_{j} \in W_{n-j}\left(\mathbb{R}^{n}\right)$, and there exist sequences

$$
\begin{array}{r}
\left\{\nu_{N}^{j}\right\}_{N=1}^{\infty} \subset C^{\infty}\left(\mathbb{R}^{n},\left|\omega_{\mathbb{R}^{n}}\right|\right), 0 \leq j \leq n, \\
\left\{B_{N}^{i j}\right\}_{N=1}^{\infty} \subset \mathcal{K}\left(\mathbb{R}^{n}\right), 1 \leq i \leq j \leq n,
\end{array}
$$

with (65) being strictly convex compact domains with smooth boundaries containing 0 at the interior such that

$\left(1_{f_{*} \psi}\right)$ for any compact subset $T \subset \mathbb{R}^{n}$, any $L \in \mathbb{N}$, and any $0 \leq j \leq n$ one has

$$
\sum_{N=1}^{\infty}\left\|\nu_{N}^{j}\right\|_{C^{L}(T)} \prod_{i=1}^{j}\left\|h_{B_{N}^{i j}}\right\|_{C^{L}\left(S^{n-1}\right)}<\infty ;
$$

$\left(2_{f_{*} \psi}\right)$ for any set $S \in \mathcal{K}\left(\mathbb{R}^{n}\right)$ and any $0 \leq j \leq n$ one has

$$
\psi_{j}(S)=\left.\sum_{N=1}^{\infty} \frac{\partial^{j}}{\partial \mu_{1} \ldots \partial \mu_{j}}\right|_{0} \nu_{N}^{j}\left(S+\sum_{i=1}^{j} \mu_{i} B_{N}^{i j}\right) .
$$


As previously the function $\nu_{N}^{j}\left(S+\sum_{i=1}^{j} \mu_{i} B_{N}^{i j}\right)$ is $C^{\infty}$-smooth in $\mu_{1}, \ldots, \mu_{j} \geq 0$ and the series (67) converges absolutely.

In [4] we have defined the product $f_{*} \phi \cdot f_{*} \psi$ as a valuation defined on convex compact subsets of $\mathbb{R}^{n}$ only by the following formula: for any $K \in \mathcal{K}\left(\mathbb{R}^{n}\right)$

$$
\begin{aligned}
\left(f_{*} \phi \cdot f_{*} \psi\right)(K)= & \sum_{j, j^{\prime}=0}^{n} \sum_{N, N^{\prime}=1}^{\infty} \frac{\partial^{j+j^{\prime}}}{\left.\partial \lambda_{1} \ldots \partial \lambda_{j} \partial \mu_{1} \ldots \partial \gamma\right|_{-}}\left(\mu_{N}^{j} \otimes \nu_{N^{\prime}}^{j^{\prime}}\right) \\
& \times\left(\Delta(K)+\left(\sum_{i=1}^{j} \lambda_{i} A_{N}^{i j} \times \sum_{i^{\prime}=1}^{j^{\prime}} \mu_{i^{\prime}} B_{N^{\prime}}^{i^{\prime} j^{\prime}}\right)\right)
\end{aligned}
$$

where $\Delta: \mathbb{R}^{n} \hookrightarrow \mathbb{R}^{n} \times \mathbb{R}^{n}$ is the diagonal imbedding, the function

$$
\left(\mu_{N}^{j} \otimes \nu_{N^{\prime}}^{j^{\prime}}\right)\left(\Delta(K)+\left(\sum_{i=1}^{j} \lambda_{i} A_{N}^{i j} \times \sum_{i^{\prime}=1}^{j^{\prime}} \mu_{i^{\prime}} B_{N^{\prime}}^{i^{\prime} j^{\prime}}\right)\right)
$$

is $C^{\infty}$-smooth in $\lambda_{1}, \ldots, \lambda_{j}, \mu_{1}, \ldots, \mu_{j^{\prime}} \geq 0$, and the series (69) converges absolutely and defines a valuation on $\mathcal{K}\left(\mathbb{R}^{n}\right)$ from the space $S V\left(\mathbb{R}^{n}\right)$. By Proposition 1.3.3 it defines a smooth valuation on $\mathcal{P}\left(\mathbb{R}^{n}\right)$. Hence we get a smooth valuation on $U \subset X$. This valuation will be denoted later on by $\left.\left.\phi\right|_{U} \cdot \psi\right|_{U}$. However this construction depends a priori on a choice of a diffeomorphism $f$ and the choices (58)-(60), (63)(65). It was however shown in [4] that once $f$ is fixed, the other choices (58)-(60), (63)-(65) do not influence the definition of $f_{*} \phi \cdot f_{*} \psi$. So let us denote temporarily the valuation we have constructed on $U$ by $\left.\left.\phi\right|_{U} \circ_{f} \psi\right|_{U}$. In order to check that the product is well defined it remains to show that if $\tilde{U} \subset X$ is another open subset and $\tilde{f}: \tilde{U} \stackrel{\sim}{\rightarrow} \mathbb{R}^{n}$ is a diffeomorphism, then

$$
\left.\left(\left.\left.\phi\right|_{U} \circ_{f} \psi\right|_{U}\right)\right|_{U \cap \tilde{U}}=\left.\left(\left.\left.\phi\right|_{\tilde{U}} \circ_{\tilde{f}} \psi\right|_{\tilde{U}}\right)\right|_{U \cap \tilde{U}}
$$

By Lemma 3.1.14 it is enough to show that for an arbitrary compact domain with smooth boundary $K \subset U \cap \tilde{U}$ such that $f(K)$ and $\tilde{f}(K)$ are convex in $\mathbb{R}^{n}$, one has

$$
\left(f_{*} \phi \cdot f_{*} \psi\right)(f(K))=\left(\tilde{f}_{*} \phi \cdot \tilde{f}_{*} \psi\right)(\tilde{f}(K)) \text {. }
$$

Let us also fix another compact domain with smooth boundary $K^{\prime} \subset U \cap \tilde{U}$ such that $K$ is contained in the interior of $K^{\prime}$. For valuations $\tilde{f}_{*} \phi$ and $\tilde{f}_{*} \psi$ we can find presentations similar to (58), (62), (63), (67). Namely

$$
\begin{aligned}
& \tilde{f}_{*} \phi=\tilde{\phi}_{0}+\cdots+\tilde{\phi}_{n}, \\
& \tilde{f}_{*} \psi=\tilde{\psi}_{0}+\cdots+\tilde{\psi}_{n},
\end{aligned}
$$

with $\tilde{\phi}_{j}, \tilde{\psi}_{j} \in W_{n-j}\left(\mathbb{R}^{n}\right)$ and there exist sequences

$$
\begin{array}{r}
\left\{\tilde{\mu}_{N}^{j}\right\}_{N=1}^{\infty},\left\{\tilde{\nu}_{N}^{j}\right\}_{N=1}^{\infty} \subset C^{\infty}\left(\mathbb{R}^{n},\left|\omega_{\mathbb{R}^{n}}\right|\right), 0 \leq j \leq n, \\
\left\{\tilde{A}_{N}^{i j}\right\}_{N=1}^{\infty},\left\{\tilde{B}_{N}^{i j}\right\}_{N=1}^{\infty} \subset \mathcal{K}\left(\mathbb{R}^{n}\right), 1 \leq i \leq j \leq n,
\end{array}
$$

with (75) being strictly convex compact domains with smooth boundaries, contain- 
ing the origin at the interior, and such that

(1) for any compact subset $T \subset \mathbb{R}^{n}$, any $L \in \mathbb{N}$, and any $0 \leq j \leq n$ one has

$$
\begin{aligned}
& \sum_{N=1}^{\infty}\left\|\tilde{\mu}_{N}^{j}\right\|_{C^{L}(T)} \prod_{i=1}^{j}\left\|h_{\tilde{A}_{N}^{i j}}\right\|_{C^{L}\left(S^{n-1}\right)}<\infty, \\
& \sum_{N=1}^{\infty}\left\|\tilde{\nu}_{N}^{j}\right\|_{C^{L}(T)} \prod_{i=1}^{j}\left\|h_{\tilde{B}_{N}^{i j}}\right\|_{C^{L}\left(S^{n-1}\right)}<\infty ;
\end{aligned}
$$

(2) for any set $S \in \mathcal{K}\left(\mathbb{R}^{n}\right)$ and any $0 \leq j \leq n$ one has

$$
\begin{aligned}
& \tilde{\phi}_{j}(S)=\left.\sum_{N=1}^{\infty} \frac{\partial^{j}}{\partial \lambda_{1} \ldots \partial \lambda_{j}}\right|_{0} \tilde{\mu}_{N}^{j}\left(S+\sum_{i=1}^{j} \lambda_{i} \tilde{A}_{N}^{i j}\right), \\
& \tilde{\psi}_{j}(S)=\left.\sum_{N=1}^{\infty} \frac{\partial^{j}}{\partial \mu_{1} \ldots \partial \mu_{j}}\right|_{0} \tilde{\nu}_{N}^{j}\left(S+\sum_{i=1}^{j} \mu_{i} \tilde{B}_{N}^{i j}\right) .
\end{aligned}
$$

We have to check that

$$
\begin{aligned}
& \sum_{j, j^{\prime}=0}^{n} \sum_{N, N^{\prime}=1}^{\infty} \frac{\partial^{j+j^{\prime}}}{\left.\partial \lambda_{1} \ldots \partial \lambda_{j} \partial \mu_{1} \ldots \partial \gamma\right|_{-}}\left(\mu_{N}^{j} \otimes \nu_{N^{\prime}}^{j^{\prime}}\right) \\
& \quad \times\left((f \times f)(K)+\left(\sum_{i=1}^{j} \lambda_{i} A_{N}^{i j} \times \sum_{i^{\prime}=1}^{j^{\prime}} \mu_{i^{\prime}} B_{N^{\prime}}^{i^{\prime} j^{\prime}}\right)\right) \\
& =\sum_{j, j^{\prime}=0}^{n} \sum_{N, N^{\prime}=1}^{\infty} \frac{\partial^{j+j^{\prime}}}{\left.\partial \lambda_{1} \ldots \partial \lambda_{j} \partial \mu_{1} \ldots \partial \gamma\right|_{-}}\left(\tilde{\mu}_{N}^{j} \otimes \tilde{\nu}_{N^{\prime}}^{j^{\prime}}\right) \\
& \quad \times\left((\tilde{f} \times \tilde{f})(K)+\left(\sum_{i=1}^{j} \lambda_{i} \tilde{A}_{N}^{i j} \times \sum_{i^{\prime}=1}^{j^{\prime}} \mu_{i^{\prime}} \tilde{B}_{N^{\prime}}^{i^{\prime} j^{\prime}}\right)\right) .
\end{aligned}
$$

We will prove the following lemma.

4.1.1. Lemma. The expression ((80)) is equal to

$$
\begin{aligned}
& \sum_{j, j^{\prime}=0}^{n} \sum_{N, N^{\prime}=1}^{\infty} \frac{\partial^{j+j^{\prime}}}{\left.\partial \lambda_{1} \ldots \partial \lambda_{j} \partial \mu_{1} \ldots \partial \gamma\right|_{-}}\left(\mu_{N}^{j} \otimes \tilde{\nu}_{N^{\prime}}^{j^{\prime}}\right) \\
& \quad \times\left((f \times \tilde{f})(K)+\left(\sum_{i=1}^{j} \lambda_{i} A_{N}^{i j} \times \sum_{i^{\prime}=1}^{j^{\prime}} \mu_{i^{\prime}} \tilde{B}_{N^{\prime}}^{i^{\prime} j^{\prime}}\right)\right) .
\end{aligned}
$$

The function $\left(\mu_{N}^{j} \otimes \tilde{\nu}_{N^{\prime}}^{j^{\prime}}\right)\left((f \times \tilde{f})(K)+\left(\sum_{i=1}^{j} \lambda_{i} A_{N}^{i j} \times \sum_{i^{\prime}=1}^{j^{\prime}} \mu_{i^{\prime}} \tilde{B}_{N^{\prime}}^{i^{\prime} j^{\prime}}\right)\right)$ in the last expression (82) is $C^{\infty}$-smooth in $0 \leq \lambda_{1}, \ldots, \lambda_{j}, \mu_{1}, \ldots, \mu_{j^{\prime}}<\varepsilon_{N}$ for some $\varepsilon_{N}>0$ depending on $K, A_{N}^{i j}, \tilde{B}_{N^{\prime}}^{i^{\prime} j^{\prime}}$, and the series converges absolutely.

Let us show first that Lemma 4.1.1 implies the equality (71) and hence implies that the product of valuations is well defined. We can apply Lemma 4.1.1 once again in a symmetric way in order to show that the expression (82) is equal to the expression (81). Thus the equality $(80)=(81)$ will be proved. 
Proof of Lemma 4.1.1. The differentiability and absolute convergence in (82) follow immediately from Proposition 3.1.13.

Let us show that $(80)=(82)$. Let us fix $j, N$. It is enough to show that

$$
\begin{aligned}
\sum_{j^{\prime}=0}^{n} \sum_{N^{\prime}=1}^{\infty} & \left.\frac{\partial^{j+j^{\prime}}}{\partial \lambda_{1} \ldots \partial \lambda_{j} \partial \mu_{1} \ldots \partial \mu_{j^{\prime}}}\right|_{0}\left(\mu_{N}^{j} \otimes \nu_{N^{\prime}}^{j^{\prime}}\right) \\
& \times\left((f \times f)(K)+\left(\sum_{i=1}^{j} \lambda_{i} A_{N}^{i j} \times \sum_{i^{\prime}=1}^{j^{\prime}} \mu_{i^{\prime}} B_{N^{\prime}}^{i^{\prime} j^{\prime}}\right)\right) \\
= & \left.\sum_{j^{\prime}=0}^{n} \sum_{N^{\prime}=1}^{\infty} \frac{\partial^{j+j^{\prime}}}{\partial \lambda_{1} \ldots \partial \lambda_{j} \partial \mu_{1} \ldots \partial \mu_{j^{\prime}}}\right|_{0}\left(\mu_{N}^{j} \otimes \tilde{\nu}_{N^{\prime}}^{j^{\prime}}\right) \\
& \times\left((f \times \tilde{f})(K)+\left(\sum_{i=1}^{j} \lambda_{i} A_{N}^{i j} \times \sum_{i^{\prime}=1}^{j^{\prime}} \mu_{i^{\prime}} \tilde{B}_{N^{\prime}}^{i^{\prime} j^{\prime}}\right)\right) .
\end{aligned}
$$

Lemma 3.1.10 implies that it is enough to show that for fixed $\lambda_{1}, \ldots, \lambda_{j}>0$ one has the equality

$$
\begin{aligned}
& \left.\sum_{j^{\prime}=0}^{n} \sum_{N^{\prime}=1}^{\infty} \frac{\partial^{j^{\prime}}}{\partial \mu_{1} \ldots \partial \mu_{j^{\prime}}}\right|_{0}\left(\mu_{N}^{j} \otimes \nu_{N^{\prime}}^{j^{\prime}}\right)\left((f \times f)(K)+\left(\sum_{i=1}^{j} \lambda_{i} A_{N}^{i j} \times \sum_{i^{\prime}=1}^{j^{\prime}} \mu_{i^{\prime}} B_{N^{\prime}}^{i^{\prime} j^{\prime}}\right)\right) \\
& \quad=\left.\sum_{j^{\prime}=0}^{n} \sum_{N^{\prime}=1}^{\infty} \frac{\partial^{j^{\prime}}}{\partial \mu_{1} \ldots \partial \mu_{j^{\prime}}}\right|_{0}\left(\mu_{N}^{j} \otimes \tilde{\nu}_{N^{\prime}}^{j^{\prime}}\right)\left((f \times \tilde{f})(K)+\left(\sum_{i=1}^{j} \lambda_{i} A_{N}^{i j} \times \sum_{i^{\prime}=1}^{j^{\prime}} \mu_{i^{\prime}} \tilde{B}_{N^{\prime}}^{i^{\prime} j^{\prime}}\right)\right) .
\end{aligned}
$$

Let us denote for brevity $A:=\sum_{i=1}^{j} \lambda_{i} A_{N}^{i j}, \mu:=\mu_{N}^{j}$. In this notation the last equality is rewritten as

$$
\begin{aligned}
& \left.\sum_{j^{\prime}=0}^{n} \sum_{N^{\prime}=1}^{\infty} \frac{\partial^{j^{\prime}}}{\partial \mu_{1} \ldots \partial \mu_{j^{\prime}}}\right|_{0}\left(\mu \otimes \nu_{N^{\prime}}^{j^{\prime}}\right)\left((f \times f)(K)+\left(A \times \sum_{i^{\prime}=1}^{j^{\prime}} \mu_{i^{\prime}} B_{N^{\prime}}^{i^{\prime} j^{\prime}}\right)\right) \\
& \quad=\left.\sum_{j^{\prime}=0}^{n} \sum_{N^{\prime}=1}^{\infty} \frac{\partial^{j^{\prime}}}{\partial \mu_{1} \ldots \partial \mu_{j^{\prime}}}\right|_{0}\left(\mu \otimes \tilde{\nu}_{N^{\prime}}^{j^{\prime}}\right)\left((f \times \tilde{f})(K)+\left(A \times \sum_{i^{\prime}=1}^{j^{\prime}} \mu_{i^{\prime}} \tilde{B}_{N^{\prime}}^{i^{\prime} j^{\prime}}\right)\right) .
\end{aligned}
$$

By Lemma 3.1.12 both sides of the last equality are equal to

$$
\int_{x \in V}\left(\left(f \circ \tilde{f}^{-1}\right)_{*} \psi\right)(f(K) \cap(x-A)) d \mu(x) .
$$

This finishes the proof of Lemma 4.1.1. Hence this also finishes the proof that the product on smooth valuations is well defined.

From the construction of the product it is easy to deduce the following result.

4.1.2. Proposition. Let $U \subset V \subset X$ be open subsets of $X$. Let $\phi, \psi \in V^{\infty}(V)$. Then

$$
\left.(\phi \cdot \psi)\right|_{U}=\left.\left.\phi\right|_{U} \cdot \psi\right|_{U}
$$

4.1.3. Theorem. The product

$$
V^{\infty}(X) \times V^{\infty}(X) \rightarrow V^{\infty}(X)
$$

is continuous, commutative, and associative. The Euler characteristic is the unit in the algebra $V^{\infty}(X)$. 
Proof. Observe first that if $X$ is diffeomorphic to $\mathbb{R}^{n}$, then this theorem was proved in [4], Theorem 4.1.2 (combined with the description of $V^{\infty}\left(\mathbb{R}^{n}\right)$ from Proposition 2.4.10 in [5]). This and Proposition 4.1.2 imply all the statements of the theorem except for continuity.

Let us prove continuity. Assume that $\phi_{N} \rightarrow \phi, \psi_{N} \rightarrow \psi$ in $V^{\infty}(X)$. We have to show that $\phi_{N} \cdot \psi_{N} \rightarrow \phi \cdot \psi$ in $V^{\infty}(X)$. Note that for any open subset $U \subset X$ diffeomeorphic to $\mathbb{R}^{n}$

$$
\left.\left.\left(\phi_{N} \cdot \psi_{N}\right)\right|_{U} \rightarrow(\phi \cdot \psi)\right|_{U} \text { in } V^{\infty}(U)
$$

by the affine case and Proposition 4.1.2.

One can easily check the following property. Let $\left\{\xi_{N}\right\} \subset V^{\infty}(X), \xi \in V^{\infty}(X)$. Let $\left\{U_{\alpha}\right\}_{\alpha}$ be an open covering of $X$. Assume that for any $\alpha$

$$
\left.\left.\xi_{N}\right|_{U_{\alpha}} \rightarrow \xi\right|_{U_{\alpha}} \text { in } V^{\infty}\left(U_{\alpha}\right) .
$$

Then $\xi_{N} \rightarrow \xi$ in $V^{\infty}(X)$. This implies the theorem.

Recall now that by [5] the assignment to any open subset $U \subset X$

$$
U \mapsto V^{\infty}(U)
$$

is a sheaf on $X$ denoted by $\mathcal{V}_{X}^{\infty}$. Proposition 4.1.2 and Theorem 4.1.3 immediately imply the following corollary.

4.1.4. Corollary. $\mathcal{V}_{X}^{\infty}$ is a sheaf of commutative associative algebras with unit (where the unit is the Euler characteristic).

\section{ACKNOWLEDGEMENTS}

The first named author is grateful to J. Bernstein for very useful discussions, and to V.D. Milman for his interest in this work and for useful discussions.

\section{REFERENCES}

[1] Alesker, Semyon; Integrals of smooth and analytic functions over Minkowski's sums of convex sets. MSRI "Convex Geometric Analysis" 34 (1998), 1-15. MR1665573 (99m:52006)

[2] Alesker, Semyon; Description of translation invariant valuations on convex sets with solution of P. McMullen's conjecture. Geom. Funct. Anal. 11 (2001), no. 2, 244-272. MR1837364 (2002e:52015)

[3] Alesker, Semyon; The multiplicative structure on polynomial continuous valuations. Geom. Funct. Anal. 14 (2004), no. 1, 1-26, also: math.MG/0301148. MR2053598 (2005d:52022)

[4] Alesker, Semyon; Theory of valuations on manifolds, I. Linear spaces. Israel J. Math., 156 (2006), 311-339. MR2282381

[5] Alesker, Semyon; Theory of valuations on manifolds, II. Adv. Math., 2007 (2006), 420-454. MR2264077

[6] Alesker, Semyon; Theory of valuations on manifolds, IV. New properties of the multiplicative structure. math.MG/0511171.

[7] Chern, S. S.; Curves and surfaces in Euclidean space. 1967 Studies in Global Geometry and Analysis, pp. 16-56. Math. Assoc. Amer. MR0212744 (35:3610)

[8] Federer, Herbert; Curvature measures. Trans. Amer. Math. Soc. 931959 418-491. MR0110078 (22:961)

[9] Federer, Herbert; Geometric measure theory. Die Grundlehren der mathematischen Wissenschaften, Band 153 Springer-Verlag New York Inc., New York 1969. MR0257325 (41:1976)

[10] Fu, Joseph H. G.; Monge-Ampère functions. I. Indiana Univ. Math. J. 38 (1989), no. 3, 745-771. MR1017333 (91d:49048)

[11] Fu, Joseph H. G.; Kinematic formulas in integral geometry. Indiana Univ. Math. J. 39 (1990), no. 4, 1115-1154. MR1087187 (92c:53043) 
[12] Fu, Joseph H. G.; Convergence of curvatures in secant approximations. J. Differential Geom. 37 (1993), no. 1, 177-190. MR1198604 (94a:53103)

[13] Fu, Joseph H. G.; Curvature measures of subanalytic sets. Amer. J. Math. 116 (1994), no. 4, 819-880. MR1287941 (95g:32016)

[14] Harvey, Reese; Lawson, H. Blaine, Jr.; Calibrated geometries. Acta Math. 148 (1982), 47-157. MR666108 (85i:53058)

[15] Kashiwara, Masaki; Schapira, Pierre; Sheaves on manifolds. With a chapter in French by Christian Houzel. Grundlehren der Mathematischen Wissenschaften [Fundamental Principles of Mathematical Sciences], 292. Springer-Verlag, Berlin, 1990. MR1074006 (92a:58132)

[16] McMullen, Peter; Valuations and dissections. Handbook of convex geometry, Vol. A, B, 933988, North-Holland, Amsterdam, 1993. MR1243000 (95f:52018)

[17] McMullen, Peter; Schneider, Rolf; Valuations on convex bodies. Convexity and its applications, 170-247, Birkhäuser, Basel, 1983. MR731112 (85e:52001)

[18] Schneider, Rolf; Convex bodies: the Brunn-Minkowski theory. Encyclopedia of Mathematics and its Applications, 44. Cambridge University Press, Cambridge, 1993. MR1216521 (94d:52007)

[19] White, Brian; A new proof of the compactness theorem for integral currents. Comment. Math. Helv. 64 (1989), no. 2, 207-220. MR997362 (90e:49052)

[20] Whitney, Hassler; Geometric integration theory. Princeton University Press, Princeton, N. J., 1957. MR0087148 (19:309c)

[21] Zähle, Martina; Approximation and characterization of generalised Lipschitz-Killing curvatures. Ann. Global Anal. Geom. 8 (1990), no. 3, 249-260. MR1089237 (91m:53055)

Department of Mathematics, Tel Aviv University, Ramat Aviv, 69978 Tel Aviv, Israel E-mail address: semyon@post.tau.ac.il

Department of Mathematics, University of Georgia, Athens, Georgia 30602

E-mail address: fu@math.uga.edu 